\title{
Intraindividuelle Variation auf lexikalisch-semantischer Ebene bei unroutinierten Schreibern des 19. Jahrhunderts*
}

\author{
Markus Schiegg und Sabrina Freund (Erlangen)
}

\begin{abstract}
Lexis and semantics are under-researched fields both in contemporary and historical sociolinguistics. This paper focuses on intra-speaker variation in texts written by unroutined writers of the 19th century and examines different types of variation in the areas of lexis and semantics. Our data are taken from a new corpus of historical patients' documents (see http://copadocs.de), which is currently being compiled as part of our research project "Flexible Writers in Language History". In order to analyse lexical-semantic variation, we combine a number of different methods. An onomasiological approach is used to explore one writer's linguistic repertoire and use of terms for women, while a structural approach allows us to compare functionally equivalent structures, such as routine formulae and expressions of a writer's origo (e.g. I, here, now). Finally, we examine the use of emotional lexis in texts by writers diagnosed with melancholia, which is found to be more frequent as compared to a control group. Our findings provide evidence for considerable intra-speaker variation in lowerclass writing. In addition, the emphasis on lexis is shown to have particular value for the investigation of patients' texts, as this approach provides insights into the stylistic repertoires of historical writers which would otherwise remain hidden.
\end{abstract}

\section{$1 \quad$ Einleitung}

Mit den Konzepten einer Sprecherdialektologie (Löffler 1986: 239) und eines flexiblen Sprechers (Macha 1991) sowie dem Vorschlag, ,die ,pragmatische Wende“ in der Linguistik für Belange der Mundartforschung nutzbar zu machen“ (Macha 2007: 317), erfolgte in der deutschsprachigen Dialektologie eine Abwendung von der traditionellen Vorstellung eines von der Sozialgruppe und Situation determinierten Sprechers. Verstärkt in den Blick genommen wurden daraufhin „Aspekte der individuellen Disposition von Sprechern“ (Macha 2005), die sich „mehr oder minder souverän in einem Möglichkeitsraum zwischen Dialekt und Standardsprache“ (Macha 1991: 2) bewegen. Diese Einsichten flossen in einige Forschungsprojekte ein, sodass individuelles Sprachhandeln mittlerweile sowohl in der ortspunktbezogenen Dialektologie (z. B. Lausberg 1993, Lenz 2003) als auch in Sprachatlanten, etwa im Norddeutschen Sprachatlas (Elmentaler/Rosenberg 2015), als zentraler Ausgangspunkt gilt.

\footnotetext{
* Diese Publikation ist im Rahmen des Elitenetzwerks Bayern entstanden und wurde aus Mitteln des Bayerischen Staatsministeriums für Wissenschaft und Kunst finanziert. Wir danken dem ärztlichen Direktor des Bezirkskrankenhauses Kaufbeuren, PD Dr. Albert Putzhammer, für seine Unterstützung bei der Arbeit mit den historischen Patientenbriefen.
} 
In der historischen (Sozio-)Linguistik dagegen nimmt intraindividuelle Variation weiterhin eine marginale Position ein. Unser Beitrag widmet sich diesem Desiderat und fokussiert auf Texte unroutinierter Schreiberinnen und Schreiber des 19. Jahrhunderts - vor allem nicht abgesendete Briefe von Patienten der süddeutschen psychiatrischen Anstalt Kaufbeuren-Irsee (vgl. Schiegg 2015). Ziel des Aufsatzes besteht darin, auszuloten, welche schriftlichen ,Möglichkeitsräume" sich bei diesen Personen zeigen - inwiefern sich also stilistische Flexibilität einerseits und externe, beeinflussende Faktoren andererseits nachweisen lassen. Wir schließen an den Sprachgeschichte von unten-Ansatz (Elspaß 2005) an, erweitern diesen aber, indem wir von Einzelschreibern und deren sprachlichen Repertoires zwischen konzeptioneller Nähe und Distanz (Koch/Oesterreicher 1985) ausgehen. Dabei bearbeiten wir die lexikalisch-semantische Ebene, die bei traditionellen soziolinguistischen Untersuchungen weniger Beachtung fand, obwohl sie bei der Ausdifferenzierung des konzeptionellen Kontinuums zentral ist (Koch/ Oesterreicher 1994: 591).

Kapitel 2 des Aufsatzes liefert einen Überblick zum Forschungsstand bezüglich intraindividueller Variation in der (historischen) Soziolinguistik mit Fokus auf der lexikalisch-semantischen Ebene. Daraufhin stellen wir in Kapitel 3 unser Korpus aus Patiententexten vor, das momentan im Rahmen der Erlanger Nachwuchsforschergruppe Flexible Schreiber in der Sprachgeschichte entsteht (s. http://copadocs.de). Kapitel 4 präsentiert die Ergebnisse unserer Analysen, wobei die ersten drei Abschnitte 4.1 bis 4.3 stilistische Flexibilität in unterschiedlichen lexikalischsemantischen Bereichen bei Einzelschreibern behandeln und Abschnitt 4.4 einen externen, beeinflussenden Faktor auf diese Flexibilität untersucht. Wir beginnen somit in Abschnitt 4.1 mit der Vorstellung des Repertoires und der großenteils flexiblen Anwendung des im Korpus hochfrequent vertretenen und stark variierenden Wortfeldes „Frauenbezeichnungen“ bei einem Schreiber mit einer relativ großen überlieferten Textmenge und damit hohem Variationspotential. Daraufhin fokussieren wir in Abschnitt 4.2 Faktoren, die Schreiber dazu bringen, sich innerhalb von Einzeltexten für bestimmte Varianten zu entscheiden. Anhand von Namen und Selbstkorrekturen führen wir hier lexikalische Variation auf textstrukturelle, funktionale und prozedurale Faktoren bei der Textproduktion zurück. Abschnitt 4.3 zeigt, wie bei Einzelschreibern lexikalische Varianten zwischen unterschiedlichen Texten variieren, wobei funktional äquivalente Strukturen sowie Origo-Bezug in Abhängigkeit vom intendierten Adressaten verglichen werden. Als beeinflussender Faktor auf sprachliche Flexibilität wird abschließend in Abschnitt 4.4 keine übliche soziolinguistische Variable wie Bildungsgrad, Geschlecht oder regionale Herkunft untersucht, sondern die Spezifik des Korpus aus Patiententexten erlaubt es, die Variable Krankheit für die Analyse der Lexik von mit „Melancholie“ diagnostizierten Patienten heranzuziehen.

\section{Forschungsstand}

\subsection{Intraindividuelle Variation in der (historischen) Soziolinguistik}

Intraindividuelle Variation erfolgt innerhalb des dem jeweiligen Sprecher zur Verfügung stehenden Repertoires sprachlicher Formen. Dies beschreibt Macha als den „Möglichkeitsraum zwischen Dialekt und Standardsprache“ (1991: 2), in dem sich der Sprecher bewegt, um seine kommunikativen Anforderungen zu erfüllen (vgl. Milroy/Milroy 1985: 119). Das jeweilige Sprachrepertoire ist aufgespannt zwischen den Polen der konzeptionellen Nähe und Distanz, 
die als ,anthropologisch begründbare, universale Kommunikationshaltungen“ (Koch/ Oesterreicher 1994: 588) zu klassifizieren sind. Das konzeptionelle Kontinuum ist dabei als eine „,von den Sprechern und Hörern aktiv gestaltete Variabilität“ (Selig 2017: 135) zu verstehen. Individuelle Variation spielte in der traditionellen Soziolinguistik eine eher marginale Rolle und wurde als eine von zahlreichen Einflussfaktoren auf Variation betrachtet (Hernández-Campoy 2016: 30). Stilistische Variation war, wie bereits Bell (1984: 145) kritisiert, „one dimension that has often been measured but seldom explained”. Heute allerdings, verstärkt durch den Einfluss der sogenannten Third Wave of Variation Studies (vgl. Eckert 2012), befindet sich stilistische Variation ,at the centre of sociolinguistic theorization and method" (Bell 2014: 297).

Dies trifft jedoch nicht für die historische Soziolinguistik zu. Auch wenn Einzelstudien zur individuellen Variation in englischen Briefen gebildeterer Schreiber des Spätmittelalters (vgl. Hernández-Campoy/García-Vidal 2018), des 16. Jahrhunders (vgl. Palander-Collin/Nevala 2011) und des 18. Jahrhunderts (vgl. Tieken-Boon van Ostade 2011) vorliegen, und Sprachrepertoires in Briefen von englischen Schreibern unterschiedlicher sozialer Herkunft aus diesem Zeitraum exemplarisch untersucht wurden (vgl. Auer 2015), so ist dennoch das Forschungsdesiderat, insbesondere in der germanistischen Sprachwissenschaft, unübersehbar. Ein Grund dafür sind sicherlich auch die bislang nicht vorhandenen Sprachkorpora, die eine Untersuchung individueller Variation von Einzelschreibern erlauben würden. Das der Studie von Elspaß (2005) zugrunde liegende und die Sprachgeschichte von unten initiierende allerdings bislang unpubliziert gebliebene - Korpus von etwa 800 Auswandererbriefen des 19 . Jahrhunderts fokussiert stärker diatopische und diachrone Ausgewogenheit denn die Möglichkeit von Untersuchungen zur stilistischen Variation. Auch anderssprachige Korpora etwa das niederländische Letters as Loot-Korpus (vgl. Rutten/Van der Wal 2014) - bestehen in der Regel aus nur einem Brieftyp, sodass etwa Registerunterschiede beim Abfassen von privaten gegenüber offiziellen Texten dort kaum untersucht werden können.

Das in der Erlanger Nachwuchsforschergruppe Flexible Schreiber in der Sprachgeschichte entstehende Korpus historischer Patientenbriefe (siehe im Detail dazu Abschnitt 3) widmet sich diesem Desiderat und integriert inbesondere Briefe von solchen Schreibern in das Korpus, von denen eine größere und vielfältige Menge an Textmaterial überliefert ist, sodass individuelle Variationsphänomene dort beobachtet werden können. Bereits durchgeführte Studien sowohl qualitativer als auch quantitativer Art zeigen, dass in diesem individuelle Variation nachgewiesen werden kann, etwa auf der Ebene der Graphematik (vgl. Schiegg 2018), der Morphologie und Syntax (vgl. Schiegg 2015) und hinsichtlich Code Switching-Phänomenen (vgl. Schiegg 2016). Die lexikalisch-semantische Ebene wurde dagegen bislang kaum beachtet.

\subsection{Die lexikalisch-semantische Ebene und intraindividuelle Variation}

Lexikalisch-semantische Aspekte spielen jedoch eine zentrale Rolle bei der Ausdifferenzierung der beiden Pole der konzeptionellen Nähe und Distanz. So ist etwa eine hohe Diversifikation des lexikalischen Materials (Type-Token-Relation) mit zahlreichen Abstrakta eher im Distanzbereich anzutreffen, geringere lexikalische Differenzierung mit einigen sogenannten Passe-Partout-Wörtern wie tun, machen und sagen sowie zahlreiche Origo-Referenzen, also nicht näher spezifizierte Bezüge zum Hier und Jetzt, dagegen im Nähebereich (Koch/ 
Oesterreicher 1994: 591). Unterschiedliche Stilebenen (gehoben, salopp, umgangssprachlich etc.) zeichnen sich ebenfalls durch spezifische Lexik und Semantik aus, die diasystematisch markiert sind (vgl. Ludwig 2009) und somit auf dem Nähe-Distanz-Kontinuum verortet werden können. Auch die Wortbildungssemantik ist maßgeblich an stilistischen Phänomenen beteiligt (vgl. Handler 2009); Hypokorismen (Kurz- und Koseformen von Namen) etwa werden zur Schaffung von Vertrautheit eingesetzt, und auch Diminutiva machen einen nähesprachlichen Stil aus. Pejorative Affigierungen, etwa mit -ling, Ge-...-e, -eln und-erei (vgl. Dammel/Quindt 2016), tragen ebenfalls zum Ausdruck einer gewissen emotionalen Haltung des Sprechers oder Schreibers bei, wobei diese sprachlichen Formen natülich immer historisiert und in ihren Gebrauchskontexten interpretiert werden müssen.

Obwohl sich die sprachlichen Ebenen der Lexik und Semantik somit dafür anbieten, intraindividuelle Variationsphänomene zu untersuchen, bilden Studien dazu in der gegenwartssprachlichen sowie historischen Soziolinguistik bislang ein Desiderat. Die internationale Soziolinguistik fokussiert traditionell phonetisch-phonologische oder morphologische Variablen, also die sogenannten ,small-scale linguistic variables“ (Bell 1984: 146). Die Lexik wurde dann vor allem hinsichlich Innovationen auf Grund von Prestige und im Kontext sozialer Netzwerke untersucht (vgl. Grzega 2012: 271). In der Germanistik hat die Wortsoziologie zwar „eine längere Tradition“ (Löffler 2016: 164), indem sie in den 1960ern aus der herkömmlichen Wortgeographie hervorgegangen ist und größere Wörterbücher immer auch stilistische Markierungen liefern, womit „Wortschatzvarianten situationell und soziolektal zugeordnet“" (Löffler 2016: 164) werden. Dennoch sind, wie Rost-Roth/Zwengel (2008: 548) festhalten, ,soziolinguistisch orientierte semantische Studien selten“.

Variationslinguistisch ist die Ebene der Lexik und Semantik oftmals nur schwer fassbar. Betrachtet man mit Labov (2004: 7) linguistische Varianten als ,,alternative ways of saying the same thing", so ist es insbesondere auf der lexikalisch-semantischen (und etwa auch auf der syntaktischen) Ebene besonders schwierig, Äquivalenz wie etwa bei phonetischen Varianten festzustellen (vgl. Hernández-Campoy 2016: 61). Deshalb wurde gefordert, so Rost-Roth/ Zwengel (2008: 549), „nicht von semantischer sondern von funktionaler Äquivalenz zu sprechen“. Methodisch umsetzen lässt sich diese Forderung, indem funktional äquivalente Strukturen gegenübergestellt und hinsichtlich ihrer individuellen lexikalisch-semantischen Ausgestaltung verglichen werden. Eine andere Möglichkeit der variationslinguistischen Untersuchung auf lexikalisch-semantischer Ebene besteht im onomasiologischen Vergleich referenzidentischer Ausdrücke. Beide Verfahren kommen in unserer empirischen Analyse in Abschnitt 4 zur Anwendung, indem etwa Ludwig F.s Ehefrau als Referenzsubjekt ausgewählt wird und die unterschiedlichen Bezeichnungen für sie - vergleichend mit anderen Frauenbezeichnungen - in den Briefen dieses Einzelschreibers und damit stilistische Variation beschrieben wird (Abschnitt 4.1). Funktional äquivalente Strukturen werden bei gleichartigen Formeln bzw. Origo-Bezügen deutlich, die aber sprachlich unterschiedlich ausgestaltet sind (Abschnitt 4.3).

\section{Datengrundlage und Untersuchungskorpus}

Datengrundlage dieses Aufsatzes bilden Texte von Patienten psychiatrischer Anstalten des 19. und frühen 20. Jahrhunderts. Deren Briefe wurden teilweise nicht abgesendet, sondern den 
Krankenakten beigelegt, wo sie - insofern die Krankenakten bis heute überliefert sind - noch immer liegen. Ein Grund dieser Briefzensur war das Ziel der Ärzte, Belege für die psychischen Krankheiten der Patienten zu sammeln, die sich in ihren Briefen manifestieren sollten. Gelegentliche Kommentare zu den Briefen in den jeweiligen Krankenakten zeugen von der Lektüre dieser durch die Ärzte. Daneben sollte verhindert werden, dass sensible Informationen über Mitpatienten oder den Anstaltsbetrieb nach außen gelangten (vgl. Scharfe 2001: 164). Teilweise wünschten auch die Angehörigen keinen weiteren Kontakt (vgl. Schiegg 2015: 171). Historische Patientenbriefe sind der medizinhistorischen Forschung bekannt, in der es im Anschluss an Porters einflussreichen Aufsatz „The Patient's View. Doing Medical History from Below" (1985) zu einem Perspektivenwechsel kam von der traditionell ärztefixierten und auf Fortschritte und Entdeckungen fokussierten Medizingeschichtsschreibung hin zu den Leidenden und deren Wahrnehmungs-, Deutungs- und Handlungsmustern. Patientenbriefe wurden daraufhin als Untersuchungsobjekte in die Studien integriert, da diese als ,einzigartige Dokumente subjektiver Wahrnehmungen von Krankheit, des Lebens in der Anstalt und der sozialen Beziehungen außerhalb der Anstalt“ (Nolte 2003: 25) gelten können. Der Sprachwissenschaft war diese Textsorte lange unbekannt, und auch der Hinweis von Riecke (2008) auf Patientenbriefe wurde nicht weiter verfolgt.

Die Erlanger Nachwuchsforschergruppe Flexible Schreiber in der Sprachgeschichte nimmt sich nun diesem Desiderat an und erstellt ein Korpus aus Patientendokumenten (s. http://copadocs.de), das am Ende über 2.000 transkribierte Texte, überwiegend Briefe, aber auch Lebensläufe und andere von den Patienten verfasste Dokumente umfassen soll. Die Texte werden nach den TEI-Richtlinien im XML-Format transkribiert, mit relevanten Metadaten angereichert und zur Nachprüfbarkeit jeweils mit Faksimiles verknüpft. Anschließend soll das Korpus online und frei verfügbar publiziert werden. Dieses ist weniger diatopisch und diachron ausgewogen als das vergleichbare Korpus historischer Auswandererbriefe (vgl. Elspaß 2005), sondern nimmt „Tiefenbohrungen“ an einzelnen Ortspunkten vor. Dabei untersuchen wir vor allem Einzelschreiber, die nur wenig Schulbildung erfahren haben und eher landwirtschaftlichen und handwerklichen Berufen nachgingen, aber dennoch mehrere Texte während der psychiatrischen Behandlung verfasst haben. Dies bietet gute Voraussetzungen zur Aufdeckung individueller Variation bei historischen Schreibern. Bislang wurden überwiegend Texte der süddeutschen psychiatrischen Anstalt Kaufbeuren-Irsee erfasst - über 10.000 Patientenbriefe wurden dort entdeckt -, in kleinerem Umfang ebenfalls aus Hamburg, Erlangen, Ansbach und Mainkofen. ${ }^{1}$ Die Überlieferung von Patientenbriefen aus den genannten Orten wird in der Forschung, soweit ersichtlich, bislang nicht erwähnt, was auf unzählige noch unbekannte und unerhobene Quellen in einigen weiteren Archiven hindeutet.

Zum Untersuchungszeitpunkt (Ende 2018) umfasste das Korpus etwa 750.000 Wörter von gut 200 unterschiedlichen Schreibern. Die Anzahl der Briefe variiert dabei zwischen einem und über 50. Für die Untersuchung ausgewählt wurde für Abschnitt 4.1 ein Schreiber mit einer Vielzahl an Texten und recht großen Textmenge (33 Briefe und ein längerer Kriegsbericht, insgesamt ca. 13.000 Wörter), um damit einen breiten Einblick in sein Repertoire zu geben und

\footnotetext{
1 Den Hinweis auf Patientenbriefe aus der Anstalt Hamburg-Langenhorn, im Staatsarchiv Hamburg aufbewahrt, verdanken wir Dr. Monika Ankele (Universitätsklinikum Hamburg-Eppendorf).
} 
das flexible Navigieren im Wortfeld „Frauenbezeichnungen“ untersuchen zu können. In den Abschnitten 4.2 und 4.3 wurden solche Schreiber herangezogen, bei denen sich die ausgewählten Phänomene exemplarisch illustrieren lassen. ${ }^{2}$ Für Abschnitt 4.4 wurden zwei Subkorpora gebildet (Melancholie-Korpus und Kontrollkorpus), die dort näher beschrieben werden.

\section{$4 \quad$ Analysen}

\subsection{Repertoire-Analyse: Frauenbezeichnungen beim Gast- und Landwirt Ludwig F.}

Die zur Untersuchung der Variation in Frauenbezeichnungen herangezogenen Briefe stammen vom Gast- und Landwirt Ludwig F. (Aktennummer 2087), geboren 1875 und beheimatet im westallgäuer Heimenkirch, einer Ortschaft etwa 20 Kilometer östlich von Lindau am Bodensee. ${ }^{3}$ Er verbrachte in den Jahren 1900, 1923 und 1926 jeweils wenige Monate in der Heil- und Pflegeanstalt Kaufbeuren-Irsee, ab Januar 1927 wurde er bis zu seinem Tod im Dezember 1935 dort stationär behandelt. Er wird in seiner Krankengeschichte beschrieben als „meistens aufgeregt, [er] halluzinirt sichtlich sehr lebhaft, ist völlig unzugänglich und verwirrt“ (02.06.1900). Metalinguistisch interessant ist der Kommentar der Krankengeschichte über seine niederalemannische Sprache, die sich vom Ostschwäbischen in Kaufbeuren (vgl. König/Renn 2007: 25) unterschied: „redet in hartem Altallgäuer Dialekt“ (01.10.1930). Im Jahre 1907 verheiratete er sich mit Maria E.; das Ehepaar soll 5 Kinder gehabt haben. Aus der Patientenakte geht recht wenig über die Entwicklung der Beziehung von Ludwig F. zu ihr hervor. Die Ehefrau befürwortete seinen Aufenthalt in der psychiatrischen Anstalt, wie etwa bei einem Eintrag am 10.01.1927 in seiner Krankengeschichte deutlich wird, am Tag, als er nach einer fast halbjährigen Flucht aus der Anstalt „mit Rat der Frau wieder gekommen“ sei. Im Oktober desselben Jahres beantragte sie seine Entmündigung. Ludwig F. schimpfte in der Anstalt ständig über seine Frau (s. u.) und wollte sich laut Eintragung in der Krankengeschichte im Januar 1927 von ihr scheiden lassen. Bei Besuchen von dieser verhielt er sich allerdings immer „sehr anständig“ (12.09.1927); er „,[t]ut schön mit der Frau, wollte mit nach Haus fahren“"(28.04.1928).

Während die in der Krankengeschichte dokumentierte Beschwerde der Ehefrau über einen Brief von ihm (21.10.1927) die Absendung mancher seiner Briefe belegt, finden sich in seiner Krankenakte dennoch 33 zurückgehaltene Briefe und Postkarten an die unterschiedlichsten Adressaten. ${ }^{4}$ Ebenso in der Akte sind zehn Seiten eines fragmentarisch erhaltenen Berichts über seine Zeit in russischer Kriegsgefangenschaft während des Ersten Weltkriegs. Beachtlich sind

\footnotetext{
${ }^{2}$ Eine Generalisierbarkeit auf das Gesamtkorpus ist insofern nur eingeschränkt möglich, als die überlieferten Daten die Untersuchbarkeit einiger Schreiber auf bestimmte Fragestellungen hin reduzieren - liegen etwa nur konzeptionell mündliche Privatbriefe vor, so lässt sich bei diesen Schreibern nicht der potentiell vorhandene, aber auf Grund der Datenlage nicht verschriftete Pol der konzeptionellen Schriftlichkeit analysieren.

${ }^{3}$ Alle Nachnamen der Patienten wurden aus Datenschutzgründen auf den Anfangsbuchstaben gekürzt.

${ }^{4}$ Bei der offiziellen Korrespondenz handelt es sich um folgende Schreiben: 1 an Obermedizinalrat, 1 an Pfleger in Kaufbeuren (Postkarte von zu Hause), 1 an Amtsgericht Weiler Lindenberg, 1 an Bürgermeister, 1 an Prinz Ruppert, 1 an Amtsgericht Kaufbeuren, 1 an ,Frau Comerzienrat“ W., 1 an Franz K., 1 an P. P, 1 unadressiert. Bei den Privatbriefen/-postkarten um folgende: 2 an Ehefrau, 2 an Ehefrau \& Kinder/Familie, 1 an Mutter \& Ehefrau, 4 an Sohn Ludwig, 1 an Sohn Josef, 1 an Familie der Ehefrau, 1 an Schwiegermutter, 11 an weitere Bekannte, die als „Freund“ angesprochen werden und/oder geduzt werden, sodass von Privatbriefen ausgegangen wird.
} 
die vielfältigen Bezeichnungen für Frauen, die im Folgenden thematisiert werden (siehe eine Übersicht mit Frequenzen in Tabelle 1). Zur Charakterisierung der Semantik der jeweiligen Bezeichnungen wird überwiegend, aber nicht nur, auf das Deutsche Wörterbuch der Grimms (DWB) zurückgegriffen, da es detaillierte Bedeutungsangaben und historische Belegstellen liefert. 5

Ein Großteil der Frauenbezeichnungen referiert auf seine Ehefrau, an die er zwar nur fünf der überlieferten Briefe adressiert, deren Handeln und Charakter er aber in einigen anderen Briefen ebenfalls thematisiert. Beginnt man beim höchsten sprachlichen Register, so fällt auf, dass er den aus dem Französischen entlehnten Begriff Dame, ,eine angesehene, vornehme frau“ (DWB 2: 703), nicht für die Ehefrau ${ }^{6}$ verwendet, sondern nur für gesellschaftlich höherstehende Offiziersgattinnen: „Die Automobie ${ }^{7}$ fuhren in sehr mäßigen Tempo, auch waren Damen dabei. Später erzählte mir ein Pleni $^{8}$, wie ihm eine Offiziersdame, durch ihr Zureden, ihm das Leben redettete“ (Bericht Kriegsgefangenschaft, undatiert). Dies zeigt im Umkehrschluss, dass Ludwig F. seine Frau nicht zu diesen höherstehenden Personen zählt. Er bezeichnet sie jedoch ebenfalls mit diesem Lexem, allerdings mit einem ironisierenden Diminutiv, „das Dämchen“ (24.09.1927, Brief an Familie der Ehefrau), dessen abwertender Charakter sowohl durch den Inhalt der Passage - er werde ,kalt gestellt“ durch Beförderung in die Heilanstalt - als auch die nachfolgenden Adjektive, ,sehr resolut und meisterhäftig“, deutlich wird. Auch bezeichnet er sie einmal als „meine Madā"“ (29.09.1928, Brief an Freund August), womit seit dem frühen 19. Jahrhundert Mätressentum und Dekadenz konnotiert waren (vgl. Kochskämper 1993: 179). ${ }^{9}$

Die mit etwa 60 Belegen deutlich häufigste Bezeichnung einer Person weiblichen Geschlechts ist Frau. Bei Ludwig F. sind damit immer verheiratete Frauen gemeint (vgl. DWB 4: 74), unverheiratete Frauen bezeichnet er als Mädel, Madl oder direkt mit ihrem Namen (s. u.). Oftmals stehen vor Frau die Possessivpronomen meine oder seine oder das Lexem erscheint in Adressatenfunktion (z. B. „Frau Vroni L. Käsereibesitzersfrau Holzhausen“, Name gekürzt, 15.08.1929). Die qualifizierenden Adjektive sind bei seiner Ehefrau zwar in den Briefanreden stets wert und lieb (z. B. „Werte Frau!“", 25.08.1927; „Meine liebe Frau \& Familie!“, 25.04.1935), wobei es sich hier wohl um schematische Routineformeln handelt (vgl. Elspaß 2005: 160). Der Briefinhalt ist dann aber viel weniger freundlich (s. u.). Auch die positiven Adjektive gnädig und wert in den Briefen an die Ehefrau sind immer abwertend verwendet, etwa „Den der Wirt hat der gnädigen Frau [...] zu gehorchen“ (15.01.1927) oder das durch den bevormundend und herablassend wirkenden Diskursmarker weißt eingeleitete „Weißt werte

\footnotetext{
${ }^{5}$ Eine Überprüfung an einem größeren Korpus, das den gleichen Zeitraum abdeckt (z.B. DTA), wäre lohnend, war im Rahmen dieser Untersuchung allerdings nicht durchführbar.

${ }^{6}$ Der Begriff Dame wird im kompletten bisherigen Korpus der Patientenbriefe nicht als Bezeichnung für eine Ehefrau verwendet und ist somit auch bei diesem Schreiber nicht zu erwarten.

${ }^{7}$ Unsere Hervorhebungen erscheinen in Fettdruck. In lateinischer Schrift geschriebene Wörter werden kursiviert, Kurrentschrift wird durch Normalschrift wiedergegeben. Der Wechsel in lateinische Schrift ist teilweise funktionslos, oftmals zeichnet Ludwig F. damit aber auch Anreden, Fremdwörter oder anderweitig Hervorzuhebendes aus (vgl. Schiegg/Sowada 2019).

${ }^{8}$ Bei Pleni handelt es sich um das russische Wort пленный (,Gefangener'), das Ludwig F. in lateinischer Schrift phonetisch wiedergibt.

${ }^{9}$ Der Begriff Madame erscheint auch hier im Kontext von Möbeleinkäufen und Dekadenz: „Nun hat meine $M a$ dam, den Divan und die Klappsessel, umgetauscht“.
} 
Frau“"(01.03.1935). Dem gegenüber stehen tatsächlich positiv eingesetzte Adjektive, etwa für seine Schwiegermutter, im Gegensatz zu seiner Ehefrau ,noch eine richtige Frau, von klarem Urteil“" (25.04.1935, an Frau und Familie), ebenso wie die Ehefrau vor dem Krieg noch eine „vernünftige [...] Frau“ (22.05.1929, an Nachbar) gewesen sei. Bei Briefanreden an andere Personen als seine Ehefrau verwendet er auch manchmal das Adjektiv liebwert (z. B. an Frau W., 23.05.1929), was etwas positiver konnotiert zu sein scheint als einzelnes wert und lieb. Das Lexem Frau ist somit bei Ludwig F. generell ein neutraler Begriff und Bewertungen ergeben sich aus dem Kontext.

Vielfältig ist Ludwig F.s Gebrauch des Lexems Weib, das im Gegenwartsdeutschen ,in niedere Sprachschichten zurückgedrängt" (König et al. 2015: 113) ist, ehemals aber das allgemeine Wort für eine verheiratete Frau war. ${ }^{10}$ Im 19. Jahrhundert gilt, dass sich ,,in neuerer zeit“" der „unreine klang“ (DWB 28: 344) angeheftet hat, ,weib wird zur frau niederen standes“ (DWB 28: 357). Dialektal war es aber noch Ende des 20. Jahrhunderts teilweise ein „normales Wort, allmählich mit Beigeschmack", so eine der Gewährspersonen des Bayerisch-Schwäbischen Sprachatlas (SBS Bd. 7.1: 41). Karte 12 dieses Atlasbandes veranschaulicht, dass sowohl positive als auch negative Konnotationen mit Weib verbunden sein konnten. Die Konnotation von Weib kann selbst bei einem Schreiber deutlich variieren. So thematisiert Ludwig F. ein früheres, von ihm selbst als „liebevollen Brief" charakterisiertes Schreiben an die Ehefrau, in dem er diese angeblich ,als mein heißgeliebtes Weib“ (24.09.1927) betitelte - der Brief selbst ist nicht in der Akte überliefert und wurde womöglich abgesendet. Auf Grund dieser metalinguistischen Einschätzung durch Ludwig F. liegt hier ein klar positiv konnotiertes Weib vor. In seiner Kriegserzählung erscheint einmal ein nicht näher definiertes „Weib, mit einer Laterne u. tat Brot, aus dem Ofen“" (undatiert). Hier steht Weib also neutral für eine unbekannte Frau. Seine Ehefrau bezeichnet er zwar meist mit Frau, variiert aber mit Weib, teilweise innerhalb weniger Zeilen, wobei im folgenden Beispiel der direkte Kontext von Weib negativer ist als der von Frau (,Geld wegnehmen'versus ,Geld erhalten'):

(1) Gast- und Landwirt Ludwig F. (Kfb Akte 2087), an das Amtsgericht (04.10.1928):

Von 9.I.27. bis 2.8. erhielt ich von der Frau nur $75 \mathrm{dl}$ Taschengeld 8 Kühe 3 Schumpen $^{11} \mathrm{u}$. ein Roß, büßten Sie inzwischen ein. Seit Pfingsten keinen Brief mehr bekomen. Ich verdiente mit Handel oft Geld aber d. Weib wollte dieses

\footnotetext{
10 Untersuchungen des Sprachwandels von Frauenbezeichnungen nahm vor allem Kochskämper (1991, 1993, 1994, 1999) vor, die mit ihren Analysen zum Gebrauch von Personenbezeichnungen im Indogermanischen und Althochdeutschen hierfür den Grundstein gelegt hat. Erklärungsansätze zu Pejorisierungstendenzen bei Frauenbezeichnungen bietet zunächst Keller (1995, 2003), der die Pejorisierung mit Galanterie und einem damit einhergehenden Invisible Hand-Effekt erklärt. Diesen Ansatz allein betrachtet Nübling (2011) als ungenügend; sie geht zusätzlich davon aus, dass sich negative Verwendungskontexte im Zusammenhang mit der Kulturgeschichte in der Bedeutung der Frauenbezeichnungen festgesetzt haben, vor allem bei sexualisierten Lexemen wie Dirne oder Magd. Laut Kochskämper (1993: 173) wird Frau im Neuhochdeutschen als Normalbezeichnung für jede erwachsene weibliche Person verwendet, während Weib vollständig pejorisiert ist. Auch laut Nübling (2011: 347) bestehen bei den referierenden Personenbezeichnungen im Neuhochdeutschen Asymmetrien. So stehen jeweils nur die Frauenbezeichnungen als sozial degradiert eine Ebene niedriger als im Mittelhochdeutschen. Herr und Mann bezeichnen dagegen jeweils den gleichen sozialen Stand. Weib bleibt nach dem Absinken von Frau im Neuhochdeutschen nur als Geschlechtsbezeichnung im biologischen Sinne im neutralen Sprachgebrauch.

${ }^{11}$ Schumpen sind junge weibliche Rinder, ein im Allgäu verbreitetes Lexem (VALTS 4: 172f.; SBS 11: 1-3).
}

ISSN 1615-3014 
In direkter Anrede verwendet Ludwig F. zweimal das Lexem Weib und qualifiziert es mit negativen Adjektiven. In einem wütenden Brief an sie, den er schematisch mit „Werte Frau!“ einleitet, schreibt er: „Du gemeines Weib“ (25.08.1927), womit er wohl auf deren „fragwürdige sittlichkeit“ (DWB 28: 344) anspielt. Im letzten, anklagenden Brief, acht Monate vor seinem Tod, erscheint folgender Satz: „Glaubst den Du Deine Weisheit reicht bis zu den Sternen, einfältiges Wiblein“" (25.04.1935). Hier modifiziert er das Lexem mit dem Diminutiv -lein, was seine Überlegenheit ihr gegenüber unterstreicht. Besonders auffällig hier ist auch das Graphem $<\mathrm{i}>$ in Wib-, das auf ein /i:/ hindeutet und somit auf die im zentralen Alemannischen und auch in Ludwig F.s Heimatort Heimenkirch im Dialekt unterbliebene Diphthongierung der hohen Langvokale (vgl. König/Renn 2007: 35, VALTS 2: 5712). Dies ist der einzige Beleg in Ludwig F.s Texten für diese Lautung und sticht damit besonders hervor. Im Gegensatz zum heutigen Standarddeutschen, in dem Weib lediglich abwertende Verwendung zeigt (vgl. Fußnote 10), kommt das Lexem hier sehr differenziert vor, also sowohl positiv konnotiert, neutral als auch negativ. Im Vergleich zu Frau sind die Gebrauchskontexte tendentiell eher negativ, was die auch damals schon übliche pejorisierte Konnotation in der Schriftsprache reflektiert.

Oft nennt Ludwig F. seine Ehefrau bei ihrem Namen, wobei die Vollform Maria nur dreimal erscheint, einmal in einem Antrag an das Amtsgericht Kaufbeuren zur Ehescheidung (09.03.1929) und zweimal im Kontext der Briefanschrift: auf einem Briefumschlag (01.03.1935) und in einer Adresszeile (25.08.1927). Die Kurzform Mari kommt dagegen zehnmal und meist im Briefkontext vor, teilweise - wie auch in der heutigen süd- und mitteldeutschen Alltagssprache üblich ${ }^{13}$ - mit Artikel vorangestellt, zum Beispiel ,die Mari ist sehr schlau“ (24.09.1927, an Familie der Ehefrau). Auch in der Adresszeile einer mit Bleistift geschriebenen Postkarte (03.05.1929) sowie in der Anrede eines kleinformatigen, ebenfalls mit Bleistift verfassten Briefs an die Ehefrau (16.03.1927) verwendet er diese Form. Hier könnten durchaus Papierformat und Schreibinstrument die nähesprachliche Form bedingt haben. ${ }^{14}$ Auffällig ist die Diminutivform mit neutralem Artikel, die Ludwig F. einmal verwendet, um damit auf die vor seiner Verehelichung beim Klosterwirt wohnende junge Frau zu referieren: „,das Marile vom Klösterle“ (25.05.1929). ${ }^{15}$ Er erinnert sich ans frühere Einkehren und an sie: „Meist schaute die Madl oben raus“ (22.05.1929). ${ }^{16}$

\footnotetext{
12 Auch der Wenker-Bogen von Heimenkirch (Nr. 40700) zeigt diese nicht-diphthongierten Formen (z.B. Satz 4: „Is“ [,Eis’; mhd. /i:/]; Satz 6: „Fier“ [,Feuer'; mhd. /y:/, mit Entrundung]; Satz 22: „lut“ [,laut'; mhd. /u:/]); vgl. REDE: https://www.regionalsprache.de/Wenkerbogen/WenkerbogenViewer.aspx?Id=67500 [09.03.2019].

13 Siehe die Karten „Artikel + Vorname“ des Atlas zur deutschen Alltagssprache (AdA): http://www.atlas-alltagssprache.de/artikelvorname/ [09.03.2019].

14 Auch das nicht diphthongierte /i:/ in Wiblein erscheint in einer mit Bleistift geschriebenen Passage im letzten Brief vom 25.04.1935, der mit Tinte beginnt, aber in Zeile 8 in Bleistift wechselt. Gleich beim Wechsel lässt er den Längenanzeiger $<\mathrm{e}>$ in libe weg - alle ca. 40 anderen Belege dieses Lexems erscheinen bei ihm dagegen korrekt mit Längenanzeiger. Eine Untersuchung dieses möglichen Zusammenhangs von Materialität/Schreibinstrument und Nähe-/Distanzsprache erscheint vielversprechend, steht aber noch aus.

15 Die gleiche Wortbildung verwendet Ludwig F. bei „das Gretle“ (22.05.1929, an Nachbar).

16 Die Form „Madl“ (wohl ,Mädchen') ist ungewöhnlich, da sie eher in der Region zwischen dem Lech und München verbreitet ist. Für Heimenkirch wäre Mädle oder Feel als Dialektformen zu erwarten (vgl. SBS 10: 174, VALTS 4: 182). Für nicht näher spezifizierte Mädchen (Plural) verwendet Ludwig F. einmal „die Mädel““
} 
Oftmals wird aus Ludwig F.s Lexemen ein Ehebezug deutlich, wobei die Bezeichnungen bei der Rückerinnerung an seine frühe Ehezeit meist positiver besetzt sind, etwa „Lebensgefährtin“ (22.05.1929, an Nachbar), „Braut“ (24.09.1927, an Familie der Frau) und „meinen Liebchen“ (01.03.1935, an Frau und Kinder). Etwas distanzierend bezeichnet er im Kontext eines Antrags auf Ehescheidung sie als „Ehehälfte“ (09.03.1929, an das Amtsgericht); er wurde „von der holden Ehehälfte, zu Unrecht, jahrelang eckelhaft behandelt" (01.03.1935, an Frau und Kinder); „meine Holde“ (23.05.1929, an Frau W.) erscheint ebenso wie „meiner Gattin“ (ebd.) dagegen neutral bis positiv, wobei „die Ehegattin“ (25.04.1935, an Frau und Familie) wieder distanzierend ist, ${ }^{17}$ was aus dem bestimmten Artikel statt Possessivpronomen mein resultiert. Negativ konnotiert ist „das Ehegspan“(25.05.1929, diverse Adressaten), und dies wird im selben Brief nochmal durch ein abwertendes Adjektiv verstärkt: „mein unerzogenes Ehegespan“ (ebd.). Das „einen höflichen [...] klang und wert“ (DWB 5: 3155) besitzende und Distanz ausdrückende Gemahlin verwendet Ludwig F. nur für andere Ehefrauen, für die eines Schlossermeisters (29.09.1928) und für eine „Comerzienratsgemahlin“ (25.05.1929).

Stark negativ konnotiert, da pejorisiert und durch ,die zeit wieder herunter gebracht“ (DWB 4: 88) sowie zusätzlich mit abwertenden Adjektiven versehen sind schließlich „das unverschähmte Frauenzim̄er" (09.03.1929) und „eingebildetes Frauen zim̄er" (15.01.1927), beide Male in Briefen an das Amtsgericht. Auch die ,streisüchtige [sic] Xantippe“ (22.05.1929, an Nachbar), eine „bösartige, den gatten drangsalierende ehefrau“ (DWB 30: 2564), wirkt beleidigend, ebenso wie die Abwertung ihres Berufs, ihrer ,gemeinen ordinären Gesinnung wie es bei Kellnerin̄en Usus ist“ (25.08.1927, an Ehefrau). Schließlich beschimpft er sie als „eingebildeter Affe" (25.04.1935). Dass Ludwig F. noch weitere Schimpfwörter für seine Ehefrau verwendet hat als die in seinen überlieferten Texten, belegt die Krankengeschichte: „Schimpft, seine Frau, ,das Mensch' habe schon 50 Kinder gehabt. ,Alles ist erlogen, was sie schreibt.““ (26.01.1933). Diese Bezeichnung ist abwertend, kommt ,,in derber rede“ (DWB 12: 2038) vor und belegt die degradierende Funktion appellativischer Frauenbezeichnungen im Neutrum (vgl. Nübling et al. 2013: 190). Der sprachwissenschaftliche Quellenwert auch der ärztlichen Aufzeichnungen ist damit nicht zu unterschätzen.

Wie Tabelle 1 zeigt, ist Ludwig F.s verschriftetes Repertoire an Frauenbezeichnungen beachtlich groß. Er variiert bei diesen einerseits zwischen seiner Ehefrau und anderen Frauen (grau unterlegt) sowie insbesondere bei ersterer je nach Kontext; pejorisierende Bezeichnungen erscheinen nur in Bezug auf seine Ehefrau. Mit Adjektiven qualifiziert er diese Bezeichnungen oftmals ironisierend. Diminutive und dialektale Lautungen erhalten die stilistische Funktion der Verniedlichung. Ludwig F. ist damit bezüglich der verwendeten Frauenbezeichnungen als flexibler Schreiber zu charakterisieren. Dem Schreibkontext angemessen sind jedoch nicht alle der Bezeichnungen, was die beiden Frauenzimmer in den offiziellen Briefen an das Amtsgericht illustrieren. Dadurch verwundert es nicht, dass diese nicht abgesendet worden sind.

(22.05.1929, an Nachbar). Womöglich liegt hier auch eine an den Namen Marile / Marl lautlich angelehnte Form vor. Auf Grund des unsicheren Status dieses Lexems wurde es nicht in Tabelle 1 aufgenommen.

${ }^{17}$ Ludwig F. stellt hier die mangelnde Empathie der Ehefrau in den Vordergrund: „Als ich unter die Mähmaschiene griet u. Herr Direckt. Heumüller hat mehr Mitleid gehabt mit mir als die Ehegattin. O Schmerz laß nach! Schwindel ohne Ausmaß“"(25.04.1935). 


\begin{tabular}{|c|c|c|c|c|c|}
\hline & \multirow[t]{2}{*}{ pejorisierend (--) } & \multicolumn{2}{|c|}{ eher pejorisierend (-) } & \multirow[t]{2}{*}{ neutral (+/-) } & \multirow[t]{2}{*}{ positiv (+) } \\
\hline & & ironisierend & verniedlichend & & \\
\hline \multirow{2}{*}{,Frau ${ }^{6}$} & & gnädige Frau [2] & & Frau (+/-) [36] & liebwerte \\
\hline & & werte Frau [2] & & Frau (+/-) [17] & Frau [4] \\
\hline \multirow{2}{*}{,Weib 6} & gemeines & & einfältiges & Weib (-) [4] & heißgeliebtes \\
\hline & Weib [1] & & Wiblein [1] & ein Weib (+/-) [1] & Weib [1] \\
\hline ,Dame ${ }^{6}$ & & Madā̄ [1] & Dämchen [1] & & Dame [2] \\
\hline Maria & & & das Marile [1] & Maria / Mari (+) [13] & \\
\hline \multirow{2}{*}{ Ehebezug } & (unerzogenes) & holde & Liebchen [1] & Ehehälfte (-) [2] & Gemahlin [2] \\
\hline & Eheg(e)span [2] & Ehehälfte [1] & & Lebensgefährtin (+) [1] & \\
\hline \multirow{4}{*}{$\begin{array}{l}\text { Schimpf- } \\
\text { wörter }\end{array}$} & \multirow{4}{*}{\multicolumn{2}{|c|}{$\begin{array}{l}\text { das unverschähmte Frauenzim̄er [1] } \\
\text { eingebildetes Frauen zim̄er [1] } \\
\text { strei[t]süchtige Xantippe [1] } \\
\text { eingebildeter Affe [1]; ,,das Mensch“18 }\end{array}$}} & & Ehegattin (-) [1] & \\
\hline & & & & Gattin (+) [3] Gattin [1] & \\
\hline & & & & Braut (+) [1] & \\
\hline & & & & Holde (+) [1] & \\
\hline
\end{tabular}

Tabelle 1: Wortfeld ,weibliche Person' in Ludwig F.s Texten (Kfb Akte 2087) ${ }^{19}$

Diese Teiluntersuchung belegte nicht nur die variationslinguistische, sondern auch die sprachhistorische und dialektologische Relevanz der Patiententexte. So konnte die zeittypische Verwendung von Weib nachgezeichnet werden, das sowohl positiv als auch negativ konnotiert sein konnte und tendentiell nähesprachlicher als Frau verwendet wurde. Dabei konnte das nicht diphthongierte alemannisch-basisdialektale /i:/ beobachtet werden. Vom verschrifteten Text direkte Rückschlüsse auf den Dialekt zu ziehen, ist allerdings häufig problematisch, was hybride Formen illustrieren. So erscheint gerade bei Wiblein zwar im Stammvokal der basisdialektale Monophthong, das Diminutivsuffix ist hier allerdings standarddeutsch und nicht regionstypisch -le (vgl. VALTS 3, Karten 176f.).

\subsection{Individuelle Variation im Texts}

In diesem Abschnitt untersuchen wir Faktoren, die Schreiber dazu bewegen, sich innerhalb von Einzeltexten für bestimmte Varianten zu entscheiden. Anhand von Namen und Selbstkorrekturen führen wir hier lexikalische Variation auf textstrukturelle, funktionale und prozedurale Faktoren bei der Texterstellung zurück.

\subsubsection{Namen}

Briefe bestehen strukturell aus mehreren Teilen, die unterschiedliche Grade der Formalität aufweisen können, was innertextliche Variation begünstigt. Im vorigen Abschnitt wurde bereits ersichtlich, dass Ludwig F. im Kontext der Briefanschrift, also auf dem Briefumschlag und in der Adresszeile, die Vollform des Namens seiner Ehefrau Maria verwendet, im Briefkontext

\footnotetext{
18 Die Beschimpfung das Mensch kommt lediglich in den ärztlichen Aufzeichnungen als direkte Wiedergabe von Ludwig F.'s Gesprochenem vor (siehe oben).

19 Die hinter den Frauenbezeichnungen stehenden Ziffern geben die Anzahl der Belege für die einzelnen Bezeichnungen im Untersuchungskorpus, also in Ludwig F.'s Texten, an. Weiß unterlegte Felder bezeichnen die Ehefrau, grau unterlegte andere weibliche Personen. Ein (+) bzw. (-) weist auf eine eher positive bzw. negative Konnotation der Bezeichnung im verwendeten Kontext hin.
} 
sie dann aber mit der Kurzform Mari bezeichnet. Ähnliches lässt sich bei einigen weiteren Schreibern beobachten, die in bestimmten Positionen Hypokorismen (Kosewörter) und Spitznamen verwenden. Der Schneider Pius G. (Kfb Akte 936) etwa schreibt in der Adresszeile „An Josefa $K$.“ (18.01.1899, Name gekürzt), während er sie dann im Brief selbst mit „Geehrte Sephi!“ anspricht. Der Schmied Emanuel F. (Ham Akte 20206) nennt in der Adresszeile einer Postkarte seine Nichte beim vollen Namen „Adelheid F.“ (02.01.1934, Name gekürzt) und dann im Text „Mein liebes Heidelchen“.

Eine solche Variation bei Namen kann auch innerhalb von Code Switching-Passagen beobachtet werden. Eine der Funktionen von Code Switching ist die Stilisierung im Sinne einer Imitation bzw. Zitation der Sprechweise einer anderen Person (vgl. Gumperz 1982: 75f.). Dieser Fall tritt beim Fabrikarbeiter Josef R. (Kfb Akte 1342) ein, der im Kontext von Code Switching seinen Spitznamen verschriftet: „Und die Marie hat zu mir gesagt, weñ man helfen Schuh schwärzen thut in der kleine Werkstatt beim Gebhardt. Du Sepp wie macht man in der Hasengaß?“ (20.03.1922). Der Schreiber imitiert hier die Sprache der erwähnten Marie, die ihn wahrscheinlich mit seinem Spitznamen Sepp anspricht. Am Schluss desselben Briefes jedoch unterschreibt er mit seinem vollen Namen: „Es grüßt euch alle Dein Dankbarer Schwager R. Joseph." (Name gekürzt). Hier spielt für die innertextliche Variation also sowohl der Faktor Textposition als auch das Code Switching eine Rolle. Ähnlich bezeichnet der Mahlknecht Georg S. (Kfb Akte 1763) in seinem 1891 verfassten Lebenslauf die Nachbarstochter im Kontext einer Gerichtsanhörung mit vollem Namen: „die Tochter Barbara N., bezeugte nichts“ (Name gekürzt), während er an einer anderen Stelle eine Aussage ihres Vaters zitiert: „u. sagte, du wirst mit meiner Babet nicht blos beten wollen, du wirst etwas anderes schon auch thun könen". Hier ist Code Switching bei der Widergabe direkter Rede der Auslöser für die Verwendung des informellen Namens (vgl. Schiegg 2016: 69). ${ }^{20}$

\subsubsection{Selbstkorrekturen}

Individuelle Variation in Einzeltexten findet sich nicht nur an unterschiedlichen Briefpositionen, sondern auch an Einzelstellen in Form von Selbstkorrekturen. Bei dieser bewussten Form von Variation handelt es sich meist um Streichungen, Verbesserungen oder Hinzufügungen, welche die Schreiber entweder noch während des Schreibprozesses oder im Nachhinein an ihren Texten vornehmen. Auer (2008: 215) unterscheidet in ihrer Untersuchung zu Selbstkorrekturen in englischen Briefen des 18. Jahrhunderts vier Kategorien: orthographische Korrekturen, grammatische Korrekturen, Stilveränderungen (,style changes“"), bei denen sich Schreiber für eine stilistisch höhergestellte oder angemessenere Variante entscheiden, und sonstige Verbesserungen wie inhaltliche Korrekturen von Zahlen, Daten, Namen oder anderen

\footnotetext{
${ }^{20}$ In Code Switching-Passagen erscheinen nicht nur informellere Namensbezeichnungen, sondern weitere Formen nähesprachlicher Lexik. Anna K. (Kfb Akte 2585) etwa bezeichnet in ihren tagebuchartigen Aufzeichnungen Türken in direkter Rede eines Bademeisters mit „der Musel“ (23.04.1852), während sie diese sonst neutral „Türken“ nennt (vgl. Schiegg 2016: 68). Auch das Diminutivsuffix -le verwendet sie zweimal in der Form „Mutterle“ (26. \& 28.04.1852) bei der Wiedergabe direkter Rede einer Stimme in ihrem Inneren bzw. eines Kindes, während sie sonst „Mutter" schreibt.
} 
Fakten. Für die Untersuchung der lexikalisch-semantischen Variation werden nun Selbstkorrekturen aus der Kategorie „style changes“ untersucht. ${ }^{21}$ Häufig erscheinen diese bei der Korrektur von als zu unspezifisch empfundener oder diaphasisch markierter Lexik.

Da in Situationen kommunikativer Nähe bzw. konzeptioneller Mündlichkeit nach Koch/ Oesterreicher (1994: 591) der jeweilige Situations- und Wissenskontext bzw. die physische Präsenz der Gesprächspartner zur Bedeutungsgebung beitragen können, müssen dabei nicht alle Bestandteile der Kommunikation verbalisiert werden. Dies erklärt die hohe Frequenz von kognitiv leichter verfügbaren sogenannten Passe-Partout-Wörtern wie machen, tun, gehen, sagen etc. und die tendenziell geringere Varianz in der Wortwahl. Bei kommunikativer Distanz bzw. konzeptioneller Schriftlichkeit werden die durch die raum-zeitliche Distanz fehlenden außersprachlichen Kontexte und Faktoren durch eine stärkere Differenzierung der Lexik kompensiert, was eine schnelle und präzise Referenz durch die Bereitstellung spezifischer lexikalischer Einheiten ermöglicht. Dies scheint auch ungeübten Schreiberinnen und Schreibern bewusst gewesen zu sein, wenn sie ein generelles Lexem mit einem spezifischeren korrigieren. Neben dem sozialen Gefälle zwischen den Schreibern und den Adressaten spielen dabei auch Faktoren wie Medium, Intention der Schreiber, bewusste Stilisierung etc. eine Rolle für die Variation zwischen nähesprachlichen und distanzsprachlichen Formen, sodass durchaus auch in Privatbriefen Korrekturen auftreten können:

(2) Schuhmacher Josef R. (Kfb Akte 6270), an seine Mutter (01.11.1929):

Wie ich da im Frülüng naus kam, hätten Sie mir 20 M geben schenken dürfen, statt abnehmen, was ich erspart hatte.

(3) Hafnermeister Johann Z. (Kfb Akte 2105), an seinen Sohn (undatiert, ca. 1867-82):

Die jenigen müssen doch am Enden doch ihr unrech das gethan Sie an mihr ferübt haben $[\ldots]$

Der Schuhmacher Josef R. nimmt in Beispiel (2) während des Schreibens eine Streichung vor und ersetzt das unspezifische Verb geben durch schenken, das seine Aussage deutlicher macht und beispielsweise von Handlungen wie leihen abgrenzt. In Beispiel (3) streicht der Hafnermeister (veraltet für, Töpfermeister') Johann Z. ebenfalls im Schreibprozess das Passe-PartoutWort gethan und ersetzt es mit dem spezifischeren Verb ferübt (,verübt').

Eine weitere Form punktueller sprachlicher Flexibilität sind Selbstkorrekturen zur Vermeidung von Vulgarismen. Schimpfwörter gehören einer niedrigeren Stilebene an, die in Wörterbüchern oft als ,vulgär“ etc. bezeichnet wird. Da laut Koch/Oesterreicher (2007) eine Affinität zwischen nähesprachlicher oder mündlicher Kommunikation und niedriger Diaphasik sowie zwischen distanzsprachlicher oder schriftlicher Kommunikation und hoher Diaphasik besteht, kann man auch hier bei Schreibern, die sich um einen distanzsprachlichen, eher konzeptionell schriftlichen Ausdruck bemühen, beobachten, wie sie bewusste Korrekturen von Lexik aus niederen Stilebenen, die eher mit konzeptioneller Mündlichkeit assoziiert sind, vornehmen. Bei Beispiel (4) besteht eine hohe Wahrscheinlichkeit für eine solche Art der Selbstkorrektur.

(4) Söldnerstochter Genovesa K. (Kfb Akte 549), an ihre Mutter (19.03.1860):

\footnotetext{
21 Auch Fairman (2008: 205) geht auf Beispiele für diese Art von Selbstkorrekturen ein und thematisiert etwa die Veränderung von ,had“ zum stilistisch höheren Latinismus „,reced““ (,received').
} 
Dan will ich wißen ob ma mir einen Dienst weiß oder wie lange ich mich noch einsperen laßen muß es iß auch besch traurig daß solche lange Zeit Sklafe sein muß [...].

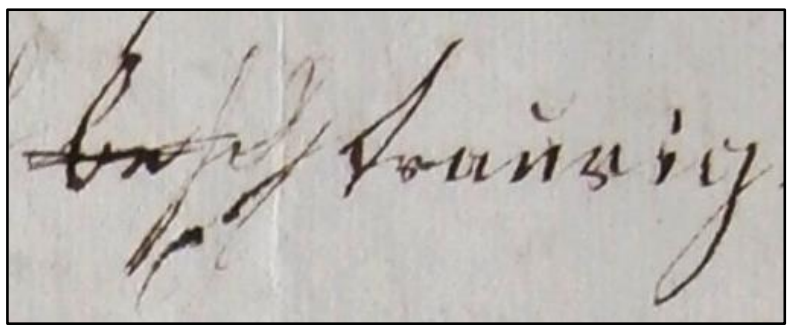

Abbildung 1: Ausschnitt aus Genovesa K.'s Brief vom 19.03.1860 (Kfb Akte 549)

Genovesa K., eine 29-jährige Söldnerstochter, beginnt hier einen mutmaßlichen Vulgarismus mit besch (...) zu verschriften. Die Strichstärke nimmt dabei ab, sodass sie sich womöglich bei der kurzen Schreibpause während des Eintauchens der Feder ins Tintenfass umentscheidet, den Wortbeginn durchstreicht und stattdessen traurig schreibt. Sofia N. liefert einen ähnlichen Beleg in Beispiel (5).

(5) Ökonomensfrau Sofia N. (Kfb Akte 1971), Erlebnisbericht (undatiert, ca. 1902-08):

[...] zuletzt kommst du noch hinter die Schweine auch wirklich als ich eines Tages furchbar schreien hörte im Schweinstall hurf sitzt er auf einem Schwein ja öfter war er darin bei ihnen.

In diesem Ausschnitt aus einem Erlebnisbericht, wie er oftmals von den Ärzten zur Selbstreflexion und weiteren Beurteilung von Krankheiten gefordert wurde (vgl. Schiegg 2016: 50), entscheidet sich die Schreiberin Sofia N., Ehefrau eines Ökonomen (veraltet für Landwirt), dessen Handeln im Textabschnitt thematisiert wird, den Vulgarismus hurt gegen das neutrale Verb sitzt auszutauschen. In dieser Situation ist der Patientin eventuell bewusst, dass die Ärzte die Qualität ihres Schreiben beurteilen, sodass sie sich bemüht, ihre Ausdrucksweise an die konzeptionelle Schriftlichkeit anzupassen.

\subsection{Individuelle Variation zwischen Texten}

Bisherige Studien zu historischen Patientenbriefen konnten belegen, dass auch unroutinierte Schreiber ihre Sprachwahl an die jeweiligen Rezipienten anpassen konnten und somit zwischen ihren Texten je nach Formalitätsgrad variierten (siehe Abschnitt 2.1). Auf lexikalisch-semantischer Ebene würden sich vergleichende Untersuchungen zur Quantität des lexikalischen Repertoires anhand von Type-Token-Relationen sowie zu dessen Qualität etwa anhand der Verwendung von Fachwortschatz und Fremdwörtern anbieten. ${ }^{22}$ Wir gehen im Folgenden jedoch exemplarisch vor und stellen zunächst einzelne funktional äquivalente, aber unterschiedlich verbalisierte Strukturen vor. Daraufhin befassen wir uns mit dem unterschiedlichen Grad von Origo-Bezug in privaten und offiziellen Briefen von zwei Schreibern.

\subsubsection{Funktional äquivalente Strukturen}

Bestimmte inhaltliche und strukturelle Elemente von Briefen wiederholen sich bei den Einzelschreibern, werden aber auf Grund der variierenden Adressaten unterschiedlich

\footnotetext{
${ }^{22}$ In Schiegg/Sowada (2019) zeigt sich etwa, dass der Schreiber Pius G. (Kfb Akte 936) nur in seinen offiziellen Briefen Code Switching ins Französische betreibt, um seine Bildung hervorzuheben.
} 
verbalisiert. So zeigt sich etwa bei der Verabschiedung häufig Variation bei Namensbezeichnungen. Die Näherin Josefa M. (Kfb Akte 2211) verabschiedet sich in mehreren Privatbriefen an ihre Schwester, indem sie sich selbst mit ihrem informelleren Namen nennt: „Deine Schwester Pepi“ (25.07.1900); in einem offiziellen Brief an den Oberarzt der psychiatrischen Anstalt verwendet sie dagegen ihren vollen Namen „Ihre ergebenste Patzientin Josepha M.“ (22.11.1911, Name gekürzt).

Häufig senden Verwandte von zu Hause Briefe sowohl an die Patienten als auch an die psychiatrische Anstalt und erkundigen sich darin nach dem Gesundheitszustand der Patienten. Dies verbalisieren sie dann oftmals unterschiedlich, wie es sich etwa beim Landwirt Josef S., Bruder von Genovesa K. (s. Beispiel 4), zeigt, der zunächt ihr selbst und später auch der Anstalt schreibt (Beispiel 6).

(6) Landwirt Joseph S. (Kfb Akte 549 [Genovesa S.]), Brief an seine Schwester ([a] 30.12.1859) und an die Anstalt ([b] 09.03.1860):

(a) $[\ldots]$ und schreibe uns auch bald wie es Dir geht.

(b) Und wir möchten auch gerne wissen, wie sich unserere Genovesa befindet, ob sie sich noch nicht gebessert hat, und bitten Euch nach Gelegenheit uns zu schreiben.

Beim direkten Vergleich der beiden funktional gleichwertigen Äußerungen erkennt man, dass die offizielle Erkundigung ausführlicher und syntaktisch komplexer ist als die private. Die Bitte an die Anstalt nach Mitteilung des Zustands der Patientin wird hier zunächst angedeutet durch die höfliche Anfrage gerne etwas wissen zu wollen, was mit dem nachvollziehbaren Wunsch nach Besserung von Genovesas Gesundheitszustand begründet wird. Im Privatbrief erscheint dabei das nähesprachliche wie es $X$ geht, während dies im offiziellen Brief mit wie sich $X$ befindet verbalisiert wird. Die Aufforderung zu schreiben selbst erscheint im Brief an seine Schwester als Imperativ „schreibe uns“, während dies im offiziellen Brief in eine Bitte gehüllt wird: „bitten Euch [...] uns zu schreiben“. Das dringlichere „bald“ im Privatbrief wird dabei durch das förmliche und distanziertere „,nach Gelegenheit“ ersetzt. Joseph S. wendet hier somit komplexe sprachliche Strategien an, um seine Aufforderung möglichst indirekt und uneindringlich zu formulieren und damit das ,negative face“ (vgl. Brown/Levinson 1987: 61) des Adressaten zu wahren.

Besonders der Vergleich von Routineformeln bietet sich an, um die unterschiedliche sprachliche Gestaltung von funktional gleichartigen Strukturen $\mathrm{zu}$ untersuchen. Unroutinierte Schreiber greifen zur Bewältigung ihrer Briefe häufig auf ein bestimmtes Grundinventar von Formeln zurück (vgl. Elspaß 2005: 164). So beginnt der Bäcker Martin O., dessen Bruder Franz sich in psychiatrischer Behandlung befindet, zwei seiner drei in der Akte überlieferten Briefe mit einer ähnlichen Formel, indem er - nach Datum und Anrede - auf ein voriges Schreiben Bezug nimmt:

(7) Bäcker Martin O. (Kfb Akte 518 [Franz O.]), an seinen Bruder ([a], 30.05. ${ }^{23}$ ) und an den Anstaltsdirektor ([b], 25.02.1859):

(a) Deinen letzten Brief hab ich richtig erhalten u. darin vernomen daß [...].

\footnotetext{
${ }^{23}$ Die Jahresangabe fehlt hier, was auf einen stärkeren Origo-Bezug im privaten als im offiziellen Brief deutet. Siehe Abschnitt 4.3.2.
} 
(b) Ihr werthes Schreiben habe ich richtig erhalten und darin vernomen daß [...].

Die Gegenüberstellung des Briefs an seinen Bruder und an den Anstaltsdirektor zeigt Unterschiede auf mehreren sprachlichen Ebenen. Orthographisch auffällig sind der fehlende Kürzungsstrich über dem $m$ von „vernomen“ in (7a) sowie das dort abgekürzte „u.“, was auf einen schnelleren Verschriftungsvorgang hindeutet. Ebenfalls erscheint dort eine Apokope in „hab“, die in der Mündlichkeit geläufig ist. Lexikalisch beachtlich ist die Variation von ,letzten Brief“ (7a) und „werthes Schreiben“ (7b), wobei Zweites im Schreiben an den Anstaltsdirektor dem formellen Distanzbereich entstammt. Dieser Befund belegt, dass selbst Routineformeln wie diese, die auch in Elspaß' (2005: 165) Auswandererkorpus in ähnlicher Form häufig beobachtet wurden, an den jeweiligen Adressaten auf vielfältige Art angepasst werden konnten. Somit ist die Aussage, dass unroutinierte Schreiber ,sich in der Verwendung von Routineformeln wenig flexibel zeigten“ (Elspaß 2005: 171) nicht uneingeschränkt gültig.

\subsubsection{Origo-Bezug}

Unterschiede zwischen privaten und offiziellen Patiententexten zeigen sich ebenfalls im Bereich des Origo-Bezugs, also des hier-jetzt-ich-Systems der subjektiven Orientierung nach Bühler (1982: 149). Das sprachliche Zeigefeld oder die Deixis beschreibt Bühler (1982: 107) als kontext- und origorelativ; so werden von der Origo des anschaulichen „Hier“ aus mit sprachlichen Mitteln alle anderen Positionen gezeigt, von der Origo „Jetzt“ aus alle anderen Zeitpunkte (Bühler 1982: 107). Nach Koch/Oesterreicher (2007: 351; 1994: 588) zeichnet sich Nähesprache bzw. (konzeptionelle) Mündlichkeit durch Situations- und Handlungseinbindung und damit eine erhöhte Frequenz von Origo-Bezügen aus. Dabei beziehen sich Sprecher oder Schreiber oft auf ihre gegenwärtige Situation in den Aspekten Zeit (jetzt, morgen), Raum (hier, $d o r t$ ), Adressat ( $d u$, Sie, Namen) und Person (ich, mein). In der Distanzsprache bzw. (konzeptionellen) Schriftlichkeit werden derartige Origo-Bezüge auf Grund der aus der raumzeitlichen Distanz resultierenden Situations- und Handlungsentbindung größtenteils vermieden. Bühler (1982: 84) spricht hier von einem „Verständnis der Rede aus den Situationsumständen“, die in der Distanzsprache nicht gegeben ist.

Bei Schreibern mit einem (vermutlich) höheren Bildungsgrad wie der Kaufmannsehegattin Rosette R. (Kfb Akte 174), die neben Gedichten in gehobener poetischer Sprache auch Briefe verfasst, kann man diese Differenzierung besonders gut erkennen.

(8) Kaufmannsehegattin Rosette R. (Kfb Akte 174), an den König ([a] 03.03.1862) und an ihren Vater ([b] 30.12.1861):

(a) An Seiner Majestät den König die unterthänigste Bitte, der Unterzeichneten durch allerhöchste Genehmigung von dem königlichen Landgericht Nördlingen das Erkenntniß ihrer Scheidungsklage rechtskräftig aussprechen, und zur Auflösung ihrer Ehe Erlaubniß ertheilen zu lassen.

(b) Deine edlen Absichten und getreue Fürsorge verstehe ich wohl und weiß sie zu würdigen, und es ist daher mein Wille, so viel in meinen Kräften steht, Deinen Befehlen zu gehorchen.

Ist das soziale Gefälle zum Adressaten sehr hoch - wie in Beispiel (8a) zum König -, vermeidet sie jede Form von Origo-Bezug. Dort verwendet sie nicht das Pronomen ich, sondern umschreibt sich selbst mit „der Unterzeichneten“. Auch ansonsten spricht sie von sich selbst in der dritten Person, etwa nicht von meiner, sondern von ,ihrer Ehe“. Für die Ansprache des 
Adressaten verwendet die Schreiberin weder die zweite noch dritte Person, sondern schreibt „An Seiner Majestät den König“, verwendet also einen „abstracten Würdenahmen“ (Adelung 1782: 684). ${ }^{24}$ Zudem finden sich in diesem Brief keine zeitlichen und räumlichen OrigoBezüge, sondern es erscheint die feste Datums- und Ortsangabe „Irrsee am $3^{\frac{\text { ten }}{}}$ März $1862^{“}$. Den Origo-Bezug hier verschriftet sie lediglich im metaphorischen Sinne innerhalb einer gereimten Passage: „Und hier auf kampfbewährtem leidensvollem Pfade“. In einem Privatbrief an ihren Vater, zu dem das soziale Gefälle geringer ist, allerdings immer noch ein asymmetrisches Kommunikationsverhältnis besteht, ergibt sich ein gegenteiliges Muster (Beispiel 8b). ${ }^{25}$ Selbst- und Adressatenbezüge werden durchgehend mit ich / mein etc. oder $d u$ / dein etc. vollzogen. Auch Zeitangaben haben in diesem Brief Origo-Bezug, so beispielsweise „Beim Rückblick auf dieses nun bald zu Ende gehende Jahr“. Die Lexik aus dem räumlichen Bereich erscheint ebenfalls immer relativ zur Origo: „befreie mich von hier“, ,,in hiesiger Erziehungs Anstalt".

Schreiber mit (vermutlich) niedrigerem Bildungsgrad wie zum Beispiel der Bäckerssohn Franz O. (Kfb Akte 518), von dessen Bruder bereits Briefe zitiert wurden (Beispiel 7), variierten teilweise auch im Origo-Bezug abhängig vom Adressaten bzw. von der Art des Briefs. In seinem offiziellen Brief an den Redakteur einer Zeitung (05.06.1853) verwendet er teilweise Pronomen wie ich, gebraucht jedoch auch Umschreibungen in der dritten Person wie „dieser Herr Sohn“ und Kombinationen aus beidem, so zum Beispiel „Ich als Unterzeichneter Baeckermeister". Adressatenbezug findet nur über die Anrede „Geehrter Herr Retackzionär"“ statt, ansonten wird gar nicht auf den Adressaten referiert. Die Zeitangaben sind ohne Verhältnis zur Origo, sondern fest: „Am Tagblat will ich lesen, am Montag den 6 Juny 1853“. Weiterhin ist in diesem offiziellen Schreiben kein hier-Bezug zu finden. Auch bei Franz O. zeigen private Briefe Lexik mit Origo-Bezug. In diesen verwendet er frequent Personalpronomen und Artikelwörter in der ersten Person und er bezieht sich direkt auf die Adressaten, etwa in einem Brief an seine Mutter und Geschwister: „Im meinem vollsten Sinne schreibe ich an Euch“ (10.08.1853). Er differenziert im selben Brief zwischen seinem Aufenthaltsort hier („hier im Narrenhaus“) und seinem früheren Zuhause $d a$ : „wo das zu Hause nie war, denn da habe ich keine ruhige Stunde gehabt“". Des Weiteren verschriftet er Zeitangaben mit Relation zur Origo, wie im Satz "Sind den heuer die Kirschen oder das Frühobst gar so theuer“ (08.08.1859, an Mutter und Schwester).

Während die bisherigen Ausführungen unterschiedliche Faktoren für sprachliche Flexibilität an Einzelschreibern belegt haben, soll der folgende Abschnitt einen Vergleich der Lexik mehrerer

\footnotetext{
${ }^{24}$ Laut Simon (2003: 106-107) veränderte sich das Anredesystem im 16. Jahrhundert dahingehend, dass für die höflichste Form der Adressatendeixis ein Pronomen verwendet wurde, dessen eigentliche Bestimmung im Paradigma der Unbeteiligten-Referenz liegt. Da in dieser Zeit die Pronomina der 2. Person Singular nicht mehr nur von gesellschaftlich Höherstehenden verwendet wurden, traten durch diesen Verlust der Exklusivität an Stelle von Ihr Personenbezeichnungen wie der Herr, bei höherstehenden Personen auch nominale Abstrakta wie Eure Majestät. Schließlich wurden diese Nominalphrasen oft durch Proformen ersetzt, sodass Pronomina der 3. Person anstelle der personenbezeichnenden Nominalphrasen traten und auch die Possessivartikel in den Phrasen selbst die 3. Person annahmen (Eure Majestät $>$ Seine Majestät) (vgl. Simon 2003: 110).

25 Dass zu ihrem Vater eine gewisse Distanz besteht, zeigt die Wendung „Deinen Befehlen zu gehorchen“ und den im späteren Briefverlauf zum Ausdruck gebrachten Wunsch „für Dich arbeiten zu dürfen“. Sie adressiert ihn zwar mit „Geliebter Papa“, degradiert sich selbst dann aber durch den Diminutiv „dein Töchterchen“.
} 
Schreiber, beeinflusst von einer externen Variable, behandeln. Die Spezifik des Korpus aus Patientenbriefen erlaubt es uns, diese Untersuchung an der Variable Krankheit durchzuführen. Die Fragestellung dabei lautet, ob eine diagnostizierte Krankheit - wir haben im Folgenden „Melancholie“ ausgewählt - Einflüsse auf den schriftlichen Sprachgebrauch von Individuen im Bereich der Lexik hat und was dies für deren sprachlichen Möglichkeitsraum bedeuten kann.

\subsection{Einflüsse der Melancholie auf die Lexik bei Einzelschreibern}

Auf Grund ihrer damaligen Bekanntheit sowie Einheitlichkeit ihrer Beschreibung und Benennung in der Forschung und der damit verbundenen guten Auffindbarkeit in den historischen Patientenakten erwies sich für unsere Untersuchung von Einflüssen psychischer Krankheiten auf die Lexik jene Krankheit als geeignet, die in der zeitgenössischen Forschung des 19. Jahrhunderts als „Melancholie“ bzw. ,melancholia“ bezeichnet wurde. Diese wird größtenteils und mit hoher Wahrscheinlichkeit heutzutage als „Depression“ nach ICD-10 (vgl. DIMDI 2019) zu den affektiven Störungen gerechnet. Unter Melancholie versteht man um die Jahrhundertwende ,ausschliesslich solche Depressionszustände, bei welchen die Gemüthsverstimmung das constante und wesentliche Moment bildet, aus welchem alle anderen Symptome (Neigung zum Suicid, Nahrungsverweigerung, Selbstanklagen u. s. w.) als Folge entspringen“ (Sommer 1901: 311). Liebmann/Edel (1903: 6-8) charakterisieren die (gesprochene) Sprache der Melancholiker als langsam und zögernd, wahrscheinlich aufgrund der Hemmung der Vorstellungstätigkeit oder Angst und Halluzinationen. Die Stimme wird als leise, monoton, singend oder klagend beschrieben. Oft würden Worte und Phrasen wiederholt. Lexikalisch relevant ist die Beobachtung, viele Melancholiker würden ihre ganze Rede auf einzelne stereotype Redensarten beschränken, sodass ihnen sogar „Ärmlichkeit des Inhaltes [ihrer] Rede“ (Liebmann/Edel 1903: 7) unterstellt wird, was auf ängstliche Ideen und Selbstvorwürfe zurückzuführen sei. Differenziertere Ergebnisse zum Gebrauch spezieller Lexik bei dieser Krankheitsform, insbesondere die höhere Frequenz von negativem Gefühlswortschatz, ergeben moderne Textanalysen etwa zum Wortgebrauch von suizidalen Menschen (vgl. Stirman/Pennebaker 2001) und zu depressiven Studenten (vgl. Rude et al. 2004, Bernard et al. 2016). ${ }^{26}$

Ausgehend von diesen Erkenntnissen soll nun untersucht werden, ob Texte von Patientinnen und Patienten aus psychiatrischen Anstalten des 19. Jahrhunderts, die als Melancholiker oder suizidal diagnostiziert wurden, im Gegensatz zu Patienten mit anderen Diagnosen bestimmte gemeinsame lexikalische Merkmale aufweisen, ob also Krankheit als eine externe Variable herangezogen werden kann, welche die sprachliche Variabilität von Einzelschreibern beeinflusst. ${ }^{27}$ Hierfür wurde aus dem Gesamtkorpus (vgl. Abschnitt 3) ein Subkorpus aus privaten und offiziellen Briefen von 10 Schreiberinnen und Schreibern erstellt, die in den Krankenakten als Melancholiker oder suizidal diagnostiziert wurden. Wichtig zur Vermeidung von zirkulären

\footnotetext{
${ }^{26}$ Generell zur Möglichkeit, mittels Textanalysen den Zusammenhang zwischen Wortwahl und psychischen Krankheiten festzustellen, sowie der Erkennbarkeit von Mustern und den eventuellen Einsatz für eine Diagnostik vgl. Pennebaker (2002).

${ }^{27}$ Hier ist anzumerken, dass selbstverständlich damalige Diagnosen nicht verifiziert werden können und dass auch der Vergleich mit heutigen Diagnosen von Depressionen eventuell nicht ganz eindeutig erfolgen kann. Es kann jedoch empirisch überprüft werden, ob diese Gruppe von Patienten tatsächlich durch den Gebrauch bestimmter Lexik von anderen Patienten unterscheidbar ist.
}

ISSN 1615-3014 
Schlüssen ist, dass nur solche Patienten für unsere Untersuchung verwendet werden, deren damalige Diagnosen nicht auf Charakteristika ihrer Schriftsprache, etwa Schriftproben, basieren, die von den Ärzten manchmal zur Klassifikation von Krankheiten herangezogen wurden (vgl. Schiegg/Thorpe 2017). Diagnosen mitsamt Krankheitsbeschreibung liegen meist in ähnlicher Form wie bei der Schmied- und Söldnersfrau Josefa P. vor:

(9) Exzerpte aus Josefa P.s Krankengeschichte (Kfb Akte 1629), Einträge ab 08.01.1907:

jam̄ert u. weint bei ihrer Aufnahme laut (08.01.1907); spricht kein Wort, starrt mit gerunzelter Stirne u. finsterer Miene vor sich hin (15.01.1907); zeigt anhaltend ein ängstliches, stuporös-negativistisches Verhalten [...]. 22.VII.1907 nach Irsee transferiert. Melancholie.

Zu dem 13.587 Tokens und 10 Schreiber umfassenden „Melancholie“-Korpus wurde eine weitere Textsammlung mit 13.221 Tokens von 11 Schreibern als Kontrollkorpus zum Vergleich angelegt. Dafür wurden jeweils Briefe von Patienten ausgewählt, die mit diversen anderen Krankheiten diagnostiziert wurden. ${ }^{28}$ Die folgenden zwei Beispiele illustrieren typische Passagen aus Patientenbriefen von Melancholikern. Dass - jedenfalls schriftsprachlich - von keiner „Ärmlichkeit des Inhaltes“ (Liebmann/Edel 1903; s. o.) die Rede sein kann, wird bereits hier deutlich.

(10) Zimmermannstochter Anna H. (Kfb Akte 120), an den Anstaltsdirektor (23.05.1856):

Ich scheine glücklich, aber der Herr der ins Verborgene sieht weiß wie viele Thränen ich geweint und hundertfacher Kumer mir am Herzen nagt. Ich glaube ${ }^{\text {nicht }}$ das Sie als rechtlicher Mañmeinen Glücke im Wege stehen und mich länger aufhalten als nöthig wäre. Und ich werde mich befleißen meine Pflichten in jeder Beziehung treulich nach zu komen. Ich möchte oft recht gerne reden und aufrichtig sein aber mir hat der Schmerz das Herz zugeschnürt.

(11) Spezereihändlerstochter Anna K. (Kfb Akte 2585), an Eltern, Freunde, Verwandte (31.05.1854):

Ich lebe theure beßte Eltern ruhig ja Oftmals unendlich glüklich weil Gott der gute Vater mich tröstet - und meine „Mutter dort", wenn auch meine Seele mein Herz manchmal wie gebrochen sind - und Sich beugen in unendlichem Jammer! „Ein“ Sonnen-Strahl v. „Dort“ in's wunde Herz und hoffend kindlich träumend hebt Sich's wieder „Empor“ glaubend fest, daß ich auch wieder die Heimath sehe denn Es kann doch nicht möglich sein, daß Sich das Herz - Sonst so besorgter - meiner redlichen, ehrlichen Ältern so lieb und gefühllos der einzigen Tochter entfremdet, weil Die ein bis Jetzt unverstandenes Herz, ein fremdes fernes Sehnen trägt - und Sonst schwieg weil des Redens Stunde immer kömmt!

Für die Untersuchung von lexikalischen Merkmalen wurden in Anlehnung an die psychologischen Studien von Rude et. al. (2004) und Stirman/Pennebaker (2001) der Gefühlswortschatz und die Wortfelder Tod und Gewalt festgesetzt. Anschließend wurden in den Patientenbriefen der Melancholiker und der Kontrollgruppe alle Wörter der folgenden Kategorien ausgezählt: In der Kategorie „Emotion negativ“ wurden Wörter aus dem Wortfeld der negativen Emotionen wie zum Beispiel Thränen oder Kum̄er (beide in Beispiel 10) gezählt. Analog dazu umfasste die Kategorie „Emotion positiv“ Wörter für positive Emotionen wie Freude oder beklücken, die auch relevant sind, da sie oft negiert vorkommen. In der Kategorie ,Innenleben neutral ${ }^{\circ}$ wurden Wörter gezählt, die das emotionale Innenleben eines Menschen beschreiben, aber we-

\footnotetext{
${ }^{28}$ Die Texte wurden jeweils für die Untersuchung bereinigt. So wurden zum Beispiel die Getrennt- und Zusammenschreibung von Komposita normalisiert oder vereinzelte Buchstaben, Zahlen etc. entfernt, um die Anzahl der Tokens nicht zu verfälschen.
} 
der negativ noch positiv konnotiert sind. Beispiele hierfür sind Herz oder Seele (beide in Beispiel 11). Wörter wie bluten, Wunde oder zerreißen, die mit (physischen) Gewaltakten assoziiert sind, wurden in die Kategorie „Gewalt“ aufgenommen. Wörter des Wortfelds Tod und Sterben wurden in der Kategorie „Tod“ gezählt, darunter getödtet, Leichenfabrik ${ }^{29}$ oder Mörder. Diese Wörter wurden zunächst pro Brief ausgezählt. Dann wurde zur Vergleichbarkeit die relative Häufigkeit von Emotionswörtern pro 100 Tokens ermittelt. Aus diesen Werten der einzelnen Briefe wurde im Anschluss der Mittelwert aller Briefe für jeden Patienten berechnet. Diese Mittelwerte veranschaulicht Abbildung 2.

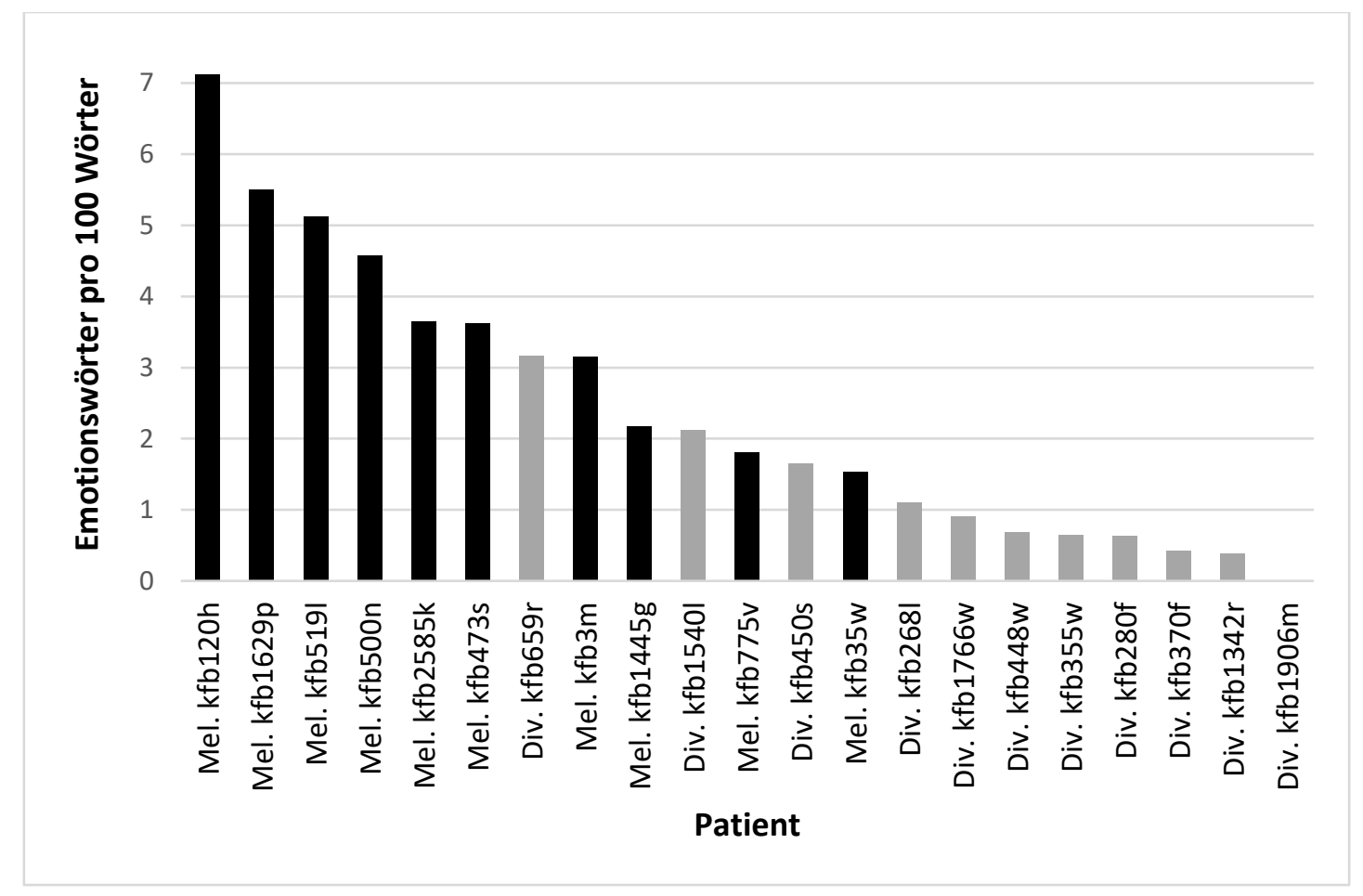

Abbildung 2: Durchschnittliche Anzahl von Emotionswörtern pro 100 Wörter $^{30}$

Es ist deutlich sichtbar, dass die Melancholiker, repräsentiert durch schwarze Balken, fast durchgehend höhere Werte aufweisen als die Kontrollgruppe (graue Balken), die nach einem Übergangsbereich sichtlich kleinere Werte zeigt. Texte von Patientinnen und Patienten, die als melancholisch oder suizidal diagnostiziert wurden, zeichnen sich also durch bestimmte gemeinsame lexikalische Merkmale aus. Die psychische Krankheit der untersuchten Schreiberinnen und Schreiber beeinflusst damit ihre Wortwahl im Bereich des Emotionswortschatzes. Wie dies in Bezug auf die sprachliche Flexibilität von Einzelschreibern interpretiert werden kann, wird im Ausblick am Ende des Aufsatzes diskutiert.

\footnotetext{
29 Dieses auffällige Kompositum stammt aus Josefa P.s Brief vom 12.08.1906 (Kfb Akte 1629; vgl. Beispiel 9).

30 Die Abkürzungen „Mel.“ und „Div.“ stehen jeweils für ,Melancholiker“ bzw. ,Diverse‘, es folgen das Archivkürzel „kfb“ (Archiv des Bezirkskrankenhauses Kaufbeuren), die Aktennummer und der erste Buchstabe des Nachnamens, um Patienten mit gleichen Aktennummern zu unterscheiden.
} 


\section{$5 \quad$ Resümee und Ausblick}

Die lexikalisch-semantische Ebene erlaubt vielfältige Möglichkeiten zur Untersuchung von intraindividueller Variation in den Texten unroutinierter Schreiber des 19. Jahrhunderts. Unterschiedliche methodische Herangehensweisen konnten in diesem Aufsatz ein breites Spektrum an Phänomenen erarbeiten. Die Zusammenstellung von Frauenbezeichnungen bei einem Gastund Landwirt belegte sowohl dessen großes Repertoire an derartigen Bezeichnungen als auch deren differenzierten Gebrauch, oftmals modifiziert durch Adjektive und Wortbildungen. Festzuhalten ist, dass auch unroutinierte Schreiber ein Bewusstsein aufweisen für unterschiedliche Grade der Formalität in Abhängigkeit von der Textstruktur (z. B. Briefumschlag vs. Briefinneres) oder insbesondere auch vom intendierten Adressaten. Je nach Adressat wurden unterschiedliche Grade von Höflichkeit bei strukturell ähnlichen Passagen sowie lexikalische Variabilität hinsichtlich des Origo-Bezugs deutlich. Textkorrekturen ließen sich als ein Bemühen um eine stärkere Differenzierung von Lexik sowie eine Vermeidung von Vulgarismen in der Schriftlichkeit deuten.

Der gezielte Einsatz adäquater sprachlicher Varianten ist jedoch durch die den jeweiligen Schreibern zur Verfügung stehenden sprachlichen Repertoires begrenzt. Diese bewegen sich, wie eingangs dargestellt, immer innerhalb von Möglichkeitsräumen, die etwa von der Schulbildung und Routine im Schreiben bestimmt werden. So lassen sich durchaus auch der Situation unangemessene Sprachformen beobachten, wie etwa die beleidigenden Frauenbezeichnungen in Ludwig F.s Brief an das Amtsgericht. Ebenso lässt sich nicht bei allen Schreibern des Korpus beispielsweise eine so klare Differenzierung des Origo-Bezugs wie in den hier präsentierten Fällen ausmachen. Dennoch ist zu konstatieren, dass die sprachlichen Differenzierungen auf der lexikalisch-semantischen Ebene vielfältig sind und an das anschließen, was auch auf anderen sprachlichen Ebenen bei unroutinierten Schreibern bereits festgestellt wurde: eine beachtliche sprachliche Flexibilität (vgl. z. B. Schiegg 2015).

Als ein in unserem Korpus zentraler Faktor, der die Möglichkeitsräume der Schreiber strukturiert, konnte deren Erkrankung herangezogen werden, und zwar der Einfluss von Melancholie auf den Sprachgebrauch: Emotionswörter erscheinen bei den daran Erkrankten fast durchgehend in größerer Häufigkeit als bei der Kontrollgruppe. Was sich in vorigen Studien an der Untersuchung von Einzelschreibern vor allem auf orthographischer Ebene andeutet (vgl. Schiegg/Thorpe 2017, Schiegg 2018), lässt sich auf der lexikalischen Ebene zunächst bestätigen: Psychische Erkrankungen können deutliche Einflüsse auf den schriftlichen Sprachgebrauch haben und verändern damit individuelle sprachliche Repertoires. Während auf der orthographischen Ebene allerdings bei zunehmender Krankheit der Verlust von Distanzsprachlichkeit und damit eine Einschränkung des Möglichkeitsraums beobachtet werden konnte, ist der Befund auf der lexikalischen Ebene differenzierter zu sehen. Eine Beeinflussung des verschrifteten sprachlichen Repertoires ist sicherlich zu bestätigen, da andere Sprachformen verbalisiert werden als bei den restlichen Schreibern und somit eine Änderung des Sprachgebrauchs angenommen werden kann. Ob aus lexikalisch-semantischer Sicht von einer Einschränkung von (schrift-)sprachlicher Kompetenz gesprochen werden kann, wie dies beim Rückgang orthographischer Fähigkeiten möglich gewesen ist, bleibt jedoch höchst fraglich; sicherlich ist von keiner Ärmlichkeit des Inhalts melancholischer (Schrift-)Sprache auszugehen, wie dies die zeitgenössische Forschung für die gesprochene Sprache tut. 
Vielmehr scheint es, als ob die Schreiber hier durch die von ihrer Krankheit hervorgerufenen Emotionen zu Ausdrucksmöglichkeiten gelangen, die sonst kaum in dieser Quantität und auch Qualität in Erscheinung treten - hier ist besonders auf die häufigen poetischen Passagen in den Texten der Melancholiker hinzuweisen, die bei unserer quantitativen Analyse im Detail nicht thematisiert werden konnten, aber Emotionen und auch Wissensbestände sichtbar machen, wie sie sonst wohl kaum verbalisiert würden. ${ }^{31}$ Weitere Untersuchungen auf lexikalisch-semantischer Ebene sowohl zur Melancholie als auch zu anderen Krankheiten wie der Paranoia, die sich laut zeitgenössischer Arbeiten durch „grosse Produktivität in der Bildung neuer Worte“ (Liebmann/Edel 1903: 30) auszeichnet, sind nun erforderlich, um unsere in diesem Bereich noch vorläufigen Ergebnisse hinsichtlich des Einflusses psychischer Krankheiten auf die verschriftete Lexik an einer breiteren Datenbasis zu validieren.

\section{Literaturverzeichnis}

\section{Primärquellen}

Ham = Staatsarchiv Hamburg, Bestand Staatskrankenanstalt Langenhorn, 352-8/7, Abl. 1995/2, Nr. 20206 (Emanuel F.).

$\mathrm{Kfb}=$ Archiv des Bezirkskrankenhauses Kaufbeuren, Patientenakten Nr. 3 (Johanna M.); 35 (Katharina W.); 120 (Anna H.); 174 (Rosette R.); 268 (Kreszenz L.); 280 (Anton F.); 355 (Maria W.); 370 (Maria F.); 448 (Leonhard W.); 450 (Magdalena S.); 473 (Josef S.); 500 (Franz N.); 518 (Franz O.); 519 (Wilhelmine von L.); 549 (Genovesa K.); 659 (Karl R.); 775 (Johann V.); 936 (Pius G.); 1342 (Josef R.); 1445 (Xaver G.); 1540 (Therese L.); 1629 (Josefa P.); 1763 (Georg S.); 1766 (Martin W.); 1906 (Karoline M.); 1971 (Sofia N.); 2087 (Ludwig F.); 2105 (Johann Z.); 2211 (Josefa M.); 2585 (Anna K.); 6270 (Josef R.).

\section{Sekundärquellen}

AdA = Elspaß, Stephan/Möller, Robert (2003ff.): Atlas zur deutschen Alltagssprache. www.atlas-alltagssprache.de [09.03.2019].

Adelung, Johann C. (1782): Umständliches Lehrgebäude der deutschen Sprache, zur Erläuterung der Deutschen Sprachlehre für Schulen. Bd. 1. Leipzig: Breitkopf.

Allioli, Joseph F. v. (1838): Die Heilige Schrift des alten und neuen Testamentes: aus der Vulgata mit Bezug auf den Grundtext neu übersetzt und mit kurzen Anmerkungen erläutert. 3., durchges. u. verb. Aufl. Bd. 3. Landshut: Palm.

Auer, Anita (2008): „, The letter which that I wrote‘: Self-Corrections in Late Modern English Letters“. In: Dossena, Marina/Tieken-Boon van Ostade, Ingrid (eds.): Studies in Late Modern English Correspondence. Methodology and Data. Frankfurt a. M. etc., Lang: 213-234. (= Linguistic Insights 76).

\footnotetext{
31 Die Zimmermannstochter Anna H. (Kfb Akte 120; Beispiel 10) etwa spricht in einer poetischen Passage über „Sensucht nach der Heimath und den Lieben“ innerhalb eines Briefs an ihre Mutter (21.10.1857) davon, dass sie „Thränenbrod gegessen“ habe, was sie vermutlich von Psalm 80,6 kennt: ,[Herr, ... wie lange wirst du ...] uns speisen mit Thränenbrod, und uns tränken mit Thränen in vollem Maße?“; zitiert nach der im 19. Jahrhundert weit verbreiteten katholischen Bibelübersetzung von Allioli (1838: 202). Das Kompositum Leichenfabrik der Melancholikerin Josefa P. (vgl. Fußnote 29) ist als besonders kreativer Sprachgebrauch zu werten.
} 
Auer, Anita (2015): „Stylistic Variation“. In: Auer, Anita/Schreier, Daniel/Watts, Richard J. (eds.): Letter Writing and Language Change. Cambridge, Cambridge Univ. Press: 133-155. https://doi.org/10.1017/CBO9781139088275.009.

Bell, Allan (1984): „Language Style as Audience Design“. Language in Society 13: 145-204. https://doi.org/10.1017/S004740450001037X.

Bell, Allan (2014): A Guidebook to Sociolinguistics. Maldan, MA: Wiley-Blackwell.

Bernard, Jared D. et al. (2016): „Depression, Language, and Affect: An Examination of the Influence of Baseline Depression and Affect Induction on Language“. Journal of Language and Social Psychology 35/3: 317-326. https://doi.org/10.1177/0261927X15589186.

Brosius, Caspar Max (1857): „Über die Sprache der Irren“. Allgemeine Zeitschrift für Psychiatrie und psychisch-gerichtliche Medizin 14: 37-64.

Brown, Penelope/Levinson, Stephen C. (1987): Politeness. Some Universals in Language Usage. Cambridge: Cambridge Univ. Press. (= Studies in Interactional Sociolinguistics 4).

Bühler, Karl (1982): Sprachtheorie. Die Darstellungsfunktion der Sprache. 2. Aufl. Stuttgart: UTB.

Dammel, Antje/Quindt, Olga (2016): „How Do Evaluative Derivational Meanings Arise? A Bit of Geforsche and Forscherei“. In: Finkbeiner, Rita/Meibauer, Jörg/Wiese, Heike (eds.): Pejoration. Amsterdam, Benjamins: 41-74. (=Linguistik Aktuell/Linguistics Today 228).

DIMDI = Deutsches Institut für Medizinische Dokumentation und Information (2019): ICD10-WHO Version 2019. Kap. 5: Psychische und Verhaltensstörungen (F00-F99). Affektive Störungen (F30-F39). https://www.dimdi.de/static/de/klassifikationen/icd/icd-10who/kode-suche/ [09.03.2019].

DWB $=$ Grimm, Jacob/Grimm, Wilhelm (eds.) (1854-1961): Deutsches Wörterbuch. 16 Bände. Leipzig: Hirzel.

Eckert, Penelope (2012): „Three Waves of Variation Study: The Emergence of Meaning in the Study of Sociolinguistic Variation“. In: Annual Review of Anthropology 41: 87-100. https://doi.org/10.1146/annurev-anthro-092611-145828.

Elmentaler, Michael/Rosenberg, Peter (2015): Norddeutscher Sprachatlas (NOSA). Bd. 1: Regiolektale Sprachlagen. Hildesheim: Olms. (= Deutsche Dialektgeographie 113/1).

Elspaß, Stephan (2005): Sprachgeschichte von unten. Untersuchungen zum geschriebenen Alltagsdeutsch im 19. Jahrhundert. Tübingen: Niemeyer. (= Reihe Germanistische Linguistik 263).

Fairman, Tony (2008): „Strike-Throughs: What Textual Alterations Can Tell us about Writers and their Scripts, 1795-1835“. In: Dossena, Marina/Tieken-Boon van Ostade, Ingrid (eds.): Studies in Late Modern English Correspondence. Methodology and Data. Frankfurt a. M. etc., Lang: 193-212. (= Linguistic Insights 76).

Grzega, Joachim (2012): „Lexical-Semantic Variables“. In: Hernández-Campoy, Juan M./Conde-Silvestre, Juan C. (eds.): The Handbook of Historical Sociolinguistics. Malden, MA, Wiley-Blackwell: 271-292.

Gumperz, John (1982): Discourse Strategies. Cambridge: Cambridge Univ. Press.

Handler, Peter (2009): „Stilistische Phänomene der Wortbildung“. In: Fix, Ulla/Gardt, Andreas/Knape, Joachim (eds.): Rhetorik und Stilistik. Ein internationales Handbuch historischer und systematischer Forschung. Bd. 2. Berlin, de Gruyter: 1563-1575. (= Handbücher zur Sprach- und Kommunikationswissenschaft 31/2). 
Hernández-Campoy, Juan M. (2016): Sociolinguistic Styles. Chichester: Wiley-Blackwell. Hernández-Campoy, Juan M./García-Vidal, Tamara (2018): „Persona Management and Identity Projection in English Medieval Society: Evidence from John Paston II“. Journal of Historical Sociolinguistics 4/1: 33-63. https://doi.org/10.1515/jhsl-2016-0027.

Keller, Rudi (1995): „Sprachwandel, ein Zerrspiegel des Kulturwandels?“ In: Lönne, KarlEgon (ed.): Kulturwandel im Spiegel des Sprachwandels. Tübingen/Basel, Francke: 207218.

Keller, Rudi (2003): Sprachwandel. Von der unsichtbaren Hand in der Sprache. 3., durchges. Aufl. Tübingen/Basel: Francke.

Koch, Peter/Oesterreicher, Wulf (1985): „Sprache der Nähe - Sprache der Distanz. Mündlichkeit und Schriftlichkeit im Spannungsfeld von Sprachtheorie und Sprachgeschichte". In: Romanistisches Jahrbuch 36: 15-43. https://doi.org/10.1515/9783110244922.15.

Koch, Peter/Oesterreicher, Wulf (1994): „Funktionale Aspekte der Schriftkultur“. In: Günther, Hartmut/Ludwig, Otto (eds.): Schrift und Schriftlichkeit/Writing and Its Use. Bd. 1. Berlin, De Gruyter: 587-604. (= Handbücher zur Sprach- und Kommunikationswissenschaft 10/1).

Koch, Peter/Oesterreicher, Wulf (2007): „Schriftlichkeit und kommunikative Distanz“. Zeitschrift für germanistische Linguistik 35/3: 346-375. https://doi.org/10.1515/zgl.2007.024.

Kochskämper, Birgit (1991): „Language History as a History of Male Language Policy. The History of German Mensch, Frau, Mann, Mädchen, Junge, Dirne... and their Indo-European Cognates“. Working Papers on Language, Gender and Sexism 2: 5-15.

Kochskämper, Birgit (1993): „Von Damen und Herren, von Männern und Frauen: Mensch und Geschlecht in der Geschichte des Deutschen“. In: Pasero, Ursula/Braun, Frederike (eds.): Frauenforschung in universitären Disziplinen: „Man räume ihnen Kanzeln und Lehrstühle ein... “. Opladen, Leske + Budrich: 153-187.

Kochskämper, Birgit (1994): „Soziales Geschlecht als Kategorie historischer Sprachwissenschaft“. In: Brandt, Gisela (ed.): Historische Soziolinguistik des Deutschen. Forschungsansätze - Korpusbildung - Fallstudien. Internationale Fachtagung. Rostock, 1.-3.9.1992. Stuttgart, Heinz: 139-149. (= Stuttgarter Arbeiten zur Germanistik 283).

Kochskämper, Birgit (1999): ,Frau' und ,Mann' im Althochdeutschen. Frankfurt a. M. etc.: Lang. (= Germanistische Arbeiten zu Sprache und Kulturgeschichte 37).

König, Werner/Elspaß, Stephan/Möller, Robert (2015): dtv-Atlas Deutsche Sprache. 18. Aufl. München: dtv.

König, Werner/Renn, Manfred (2007): Kleiner Sprachatlas von Bayerisch-Schwaben. Augsburg: Wißner.

Labov, William (2004): „Quantitative Analysis of Linguistic Variation“. In: Ammon, U1rich/Dittmar, Norbert/Mattheier, Klaus J./Trudgill, Peter (eds.): Sociolinguistics. Soziolinguistik. 2. Aufl. Bd. 1. Berlin, de Gruyter: 6-21. (= Handbücher zur Sprach- und Kommunikationswissenschaft 3/1).

Lausberg, Helmut (1993): Situative und individuelle Sprachvariation im Rheinland. Variablenbezogene Untersuchungen anhand von Tonbandaufnahmen aus Erftstadt-Erp. Köln: Böhlau.

Lenz, Alexandra (2003): Struktur und Dynamik des Substandards. Eine Studie zum Westmitteldeutschen (Wittlich/Eifel). Stuttgart: Steiner. (=ZDL-Beiheft 125). 
Liebmann, Albert/Edel, Max (1903): Die Sprache der Geisteskranken nach stenographischen Aufzeichnungen. Halle a. S.: Marhold.

Löffler, Heinrich (1986): „Sind Soziolekte neue Dialekte? Zum Aufgabenfeld einer nachsoziolinguistischen Dialektologie“. In: Schöne, Albrecht (ed.): Kontroversen, alte und neue. Akten des VII. Internationalen Germanistenkongresses. Göttingen 1985. Bd. 4. Tübingen, Niemeyer: 232-239.

Löffler, Heinrich (2016): Germanistische Soziolinguistik. 5. Aufl. Berlin: Schmidt.

Ludwig, Klaus-Dieter (2009): „Stilistische Phänomene der Lexik“. In: Fix, Ulla/Gardt, Andreas/Knape, Joachim (eds.): Rhetorik und Stilistik/Rhetorics and Stylistics. Bd. 2. Berlin, de Gruyter: 1575-1594. (= Handbücher zur Sprach- und Kommunikationswissenschaft $31 / 2$ ).

Macha, Jürgen (1991): Der flexible Sprecher. Untersuchungen zu Sprache und Sprachbewußtsein rheinischer Handwerksmeister. Köln: Böhlau.

Macha, Jürgen (2005): „Entwicklungen und Perspektiven in der Dialektologie des Deutschen: Einige Schlaglichter". Linguistik Online 24/3. https://doi.org/10.13092/lo.24.635.

Macha, Jürgen (2007): „Pragmatik und Sprachrealität. Eine dialektologische Forschungsskizze“. Niederdeutsches Wort 47: 317-326.

Milroy, James/Milroy, Lesley (1985): Authority in Language. Investigating Language Prescription and Standardization. London: Routledge.

Nolte, Karen (2003): Gelebte Hysterie. Erfahrung, Eigensinn und psychiatrische Diskurse im Anstaltsalltag um 1900. Frankfurt a. M.: Campus.

Nübling, Damaris (2011): „Von der ,Jungfrau‘ zur ,Magd‘, vom ,Mädchen“ zur ,Prostituierten': Die Pejorisierung der Frauenbezeichnungen als Zerrspiegel der Kultur und als Effekt männlicher Galanterie?“ In: Riecke, Jörg (ed.): Historische Semantik. Berlin, de Gruyter: 344-359. (= Jahrbuch für germanistische Sprachgeschichte 2).

Nübling, Damaris/Busley, Simone/Drenda, Juliane (2013): „Dat Anna und s Eva - Neutrale Frauenrufnamen in deutschen Dialekten und im Luxemburgischen zwischen pragmatischer und semantischer Genuszuweisung“. Zeitschrift für Dialektologie und Linguistik 53/2: 152196. https://www.jstor.org/stable/24548013.

Palander-Collin, Minna/Nevala, Minna (2011): „Sociopragmatic Aspects of Person Reference in Nathaniel Bacon's Letters“. In: Pahta, Päivi/Jucker, Andreas (eds.): Communicating Early English Manuscripts. Cambridge, Cambridge Univ. Press: 102-117.

Pennebaker, James W. (2002): ,What Our Words Can Say about Us: Towards a Broader Language Psychology“. Psychological Science Agenda 15: 8-9.

Porter, Roy (1985): „The Patient's View. Doing Medical History from Below“. Theory and Society 14/2: 175-198. https://www.jstor.org/stable/657089.

$\mathrm{REDE}=\mathrm{Schmidt}$, Jürgen E./Herrgen, Joachim/Kehrein, Roland (eds.): REDE. regionalsprache.de. Marburg: Deutscher Sprachatlas. https://regionalsprache.de [09.03.2019].

Riecke, Jörg (2008): „Laien-Texte in psychiatrischen Krankenakten. Ein Beitrag zum Sprachgebrauch ,kleiner Leute“ am Beginn des 20. Jahrhunderts“. Dituria. Zeitschrift für Germanistische Sprach- und Literaturwissenschaft 4: 46-56.

Rost-Roth, Martina/Zwengel, Almut (2008): „Semantik/Semantics“. In: Ammon, Ulrich/Dittmar, Norbert/Mattheier, Klaus J./Trudgill, Peter (eds.): Sociolinguistics/Soziolinguistik. 2. 
Aufl. Bd. 1. Berlin, de Gruyter: 546-555. (= Handbücher zur Sprach- und Kommunikationswissenschaft 3/1).

Rude, Stephanie/Gortner, Eva-Maria/Pennebaker, James W. (2004): „Language Use of Depressed and Depression-Vulnerable College Students“. Cognition and Emotion 18/8: 11211133. https://doi.org/10.1080/02699930441000030.

Rutten, Gijsbert/Van der Wal, Marijke J. (2014): Letters as Loot. A Sociolinguistic Approach to Seventeenth- and Eighteenth-Century Dutch. Amsterdam/Philadelphia: John Benjamins. (=Advances in Historical Sociolinguistics 2).

SBS = König, Werner (ed.) (1997-2009): Sprachatlas von Bayerisch-Schwaben. 14 Bände. Heidelberg: Winter.

Scharfe, Martin (2001): „Briefe aus dem Irrenhaus. Selbstzeugnisse von Patientinnen aus der Frühzeit der Marburger Anstalt“. In: Sandner, Peter/Aumüller, Gerhard/Vanja, Christina (eds.): Heilbar und nützlich. Ziele und Wege der Psychiatrie in Marburg an der Lahn. Marburg, Jonas: 163-183.

Schiegg, Markus (2015): „Der flexible Schreiber in der Sprachgeschichte. Grammatische Variation in süddeutschen Patientenbriefen des 19. Jahrhunderts". Zeitschrift für Dialektologie und Linguistik 82/2: 169-205. https://www.jstor.org/stable/24770223.

Schiegg, Markus (2016): „Code Switching in Lower-Class Writing. Autobiographies by Patients from Southern German Psychiatric Hospitals (1852-1931)“. Journal of Historical Sociolinguistics 2/1: 47-81. https://doi.org/10.1515/jhsl-2016-0003.

Schiegg, Markus (2018): „Factors of Intra-Speaker Variation in Nineteenth-Century LowerClass Writing“. Neuphilologische Mitteilungen 119: 101-120.

Schiegg, Markus/Sowada, Lena (2019): „Script Switching in Nineteenth-Century Lower-Class German Handwriting“. In: Paedagogica Historica. https://doi.org/10.1080/00309230.2019.1622574

Schiegg, Markus/Thorpe, Deborah (2017): „Historical Analyses of Disordered Handwriting. Perspectives on Early 20th-Century Material from a German Psychiatric Hospital." Written Communication 34/1: 30-53. https://doi.org/10.1177/0741088316681988.

Selig, Maria (2017): „Plädoyer für einen einheitlichen, aber nicht einförmigen Sprachbegriff: Zur aktuellen Rezeption des Nähe-Distanz-Modells“. Romanistisches Jahrbuch 68: 114145. https://doi.org/10.1515/roja-2017-0005.

Simon, Horst J. (2003): Für eine grammatische Kategorie «Respekt» im Deutschen. Synchronie, Diachronie und Typologie der deutschen Anredepronomina. Tübingen: Niemeyer.

Sommer, Robert (1901): Diagnostik der Geisteskrankheiten für praktische Ärzte und Studierende. 2., umgearb. und verm. Aufl. Berlin: Urban \& Schwarzenberg.

Stirman, Shannon W./Pennebaker, James W. (2001): „Word Use in the Poetry of Suicidal and Nonsuicidal Poets“. Psychosomatic Medicine 63/4: 517-522.

Tieken-Boon van Ostade, Ingrid (2011): The Bishop's Grammar. Robert Lowth and the Rise of Prescriptivism. Oxford: Oxford Univer. Press.

VALTS = Gabriel, Eugen (ed.) (1985-2006): Vorarlberger Sprachatlas mit Einschluss des Fürstentums Liechtenstein, Westtirols und des Allgäus (VALTS). 5 Bände. Bregenz: Vorarlberger Landesregierung. 